\title{
Validating and modeling the impact of high- frequency rapid antigen screening on COVID-19 spread and outcomes
}

Beatrice Nash

E25Bio, Inc.

Anthony Badea

E25Bio, Inc.

Ankita Reddy

E25Bio, Inc.

Miguel Bosch

E25Bio, Inc.

Nol Salcedo

E25Bio, Inc.

Adam Gomez

E25Bio, Inc.

Alice Versiani

Universidade Federal de Minas Gerais

Gislaine Dutra

Faculdade de Medicina de São José do Rio Preto (FAMERP)

Thayza dos Santos

Faculdade de Medicina de São José do Rio Preto (FAMERP)

Bruno Milhim

Faculdade de Medicina de São José do Rio Preto (FAMERP)

Marilia Moraes

Faculdade de Medicina de São José do Rio Preto (FAMERP)

Guilherme Campos

Faculdade de Medicina de São José do Rio Preto (FAMERP)

Flávia Quieroz

Faculdade de Medicina de São José do Rio Preto (FAMERP)

Andreia Francesli Negri Reis

Prefeitura Municipal de Sao Jose do Rlo Preto

Mauricio Nogueira

Faculdade de Medicina de São José do Rio Preto https://orcid.org/0000-0003-1102-2419 
Tufts University Friedman School Nutrition Science and Policy https://orcid.org/0000-0002-9562-4734 Irene Bosch

E25Bio

Bobby Brooke Herrera ( $\square$ bbherrera@e25bio.com )

E25Bio, Inc.

Article

Keywords: Computational Modeling, Quantitative Real-time Polymerase Chain Reaction, Antigen-based Surveillance, Societal Re-opening

Posted Date: November 16th, 2020

DOl: https://doi.org/10.21203/rs.3.rs-104765/v1

License: (c) (i) This work is licensed under a Creative Commons Attribution 4.0 International License. Read Full License 
1 Validating and modeling the impact of high-frequency rapid antigen screening on COVID-

219 spread and outcomes

3

4 Authors: Beatrice Nash ${ }^{1,2 *}$, Anthony Badea ${ }^{1,3^{*}}$, Ankita Reddy ${ }^{1,4^{*}}$, Miguel Bosch $^{1,5}$, Nol Salcedo ${ }^{1}$,

5 Adam R. Gomez ${ }^{1}$, Alice Versiani ${ }^{6}$, Gislaine Celestino Dutra Silva ${ }^{6}$, Thayza Maria Izabel Lopes

6 dos Santos $^{6}$, Bruno H. G. A. Milhim ${ }^{6}$, Marilia M Moraes ${ }^{6}$, Guilherme Rodrigues Fernandes

7 Campos $^{6}$, Flávia Quieroz ${ }^{6}$, Andreia Francesli Negri Reis ${ }^{6}$, Mauricio L. Nogueira ${ }^{6}$, Elena N.

8 Naumova $^{7}$, Irene Bosch ${ }^{1,8}$, Bobby Brooke Herrera $^{1,9 \dagger}$

9

10 *These authors contributed equally to this work.

12 Affiliations:

$13 \quad{ }^{1}$ E25Bio, Inc., Cambridge, MA, USA

$14{ }^{2}$ Department of Computer Science, Harvard University School of Engineering and Applied

15 Sciences, Cambridge, MA, USA

$16{ }^{3}$ Department of Physics, Harvard University, Cambridge, MA, USA

$17{ }^{4}$ Perelman School of Medicine, University of Pennsylvania, Philadelphia, PA, USA

$18{ }^{5}$ InfoGeosciences LLC, Houston, TX, USA

$19{ }^{6}$ Faculdade de Medicina de São José do Rio Preto (FAMERP), São José do Rio Preto, Brazil

$20{ }^{7}$ Division of the Nutrition Epidemiology and Data Science, Friedman School of Nutrition Science

21 and Policy, Tufts University, Boston, MA, USA

$22{ }^{8}$ Department of Medicine, Mount Sinai School of Medicine, New York, NY, USA 
$23{ }^{9}$ Department of Immunology and Infectious Diseases, Harvard T.H. Chan School of Public Health,

24 Boston, MA, USA

25

26 Corresponding Author. BBH, email: bbherrera@e25bio.com

27

28 Short Title: Modeling rapid antigen testing on COVID-19 spread

29

30

31

32

33

34

35

36

37

38

39

40

41

42

43

44

45 


\section{Abstract}

High frequency screening of populations has been proposed as a strategy in facilitating

48 control of the COVID-19 pandemic. We use computational modeling, coupled with clinical data

49 from rapid antigen tests, to predict the impact of frequent viral antigen rapid testing on COVID-

5019 spread and outcomes. Using patient nasal or nasopharyngeal swab specimens, we demonstrate

51 that the sensitivity/specificity of two rapid antigen tests compared to quantitative real-time

52 polymerase chain reaction (qRT-PCR) are $80.0 \% / 91.1 \%$ and $84.7 \% / 85.7 \%$, respectively;

53 moreover, sensitivity correlates directly with viral load. Based on COVID-19 data from three

54 regions in the United States and São José do Rio Preto, Brazil, we show that high frequency,

55 strategic population-wide rapid testing, even at varied accuracy levels, diminishes COVID-19

56 infections, hospitalizations, and deaths at a fraction of the cost of nucleic acid detection via qRT-

57 PCR. We propose large-scale antigen-based surveillance as a viable strategy to control SARS-

58 CoV-2 spread and to enable societal re-opening.

59

60

61

62

63

64

65

66

67

68 


\section{INTRODUCTION}

The COVID-19 pandemic has taken an unprecedented toll on lives, wellbeing, healthcare

71 systems, and global economies. As of 4 November 2020, there have been more than 47.6 million

72 confirmed cases globally with more than 1 million confirmed deaths ${ }^{1}$. However, these numbers

73 and the current mapping of disease spread present an incomplete picture of the outbreak largely

74 due to the lack of adequate testing, particularly as undetected infected cases are the main source

75 of disease spread ${ }^{2-7}$. It is estimated that the reported detection rate of actual COVID-19 cases is

76 only $1-2 \%{ }^{5}$. As of November 2020, the United States, Brazil, and India remain the top three

77 countries with the highest number of COVID-19 cases and deaths worldwide. As countries begin

78 to re-open their economies, a method for accessible and frequent surveillance of COVID-19, with

79 the necessary rapid quarantine measures, is crucial to prevent the multiple resurgences of the 80 disease.

81 The current standard of care rightfully places a strong focus on the diagnostic limit of

82 detection, yet frequently at the expense of both cost and turnaround time. This situation has

83 contributed to limited population testing largely due to a dearth of diagnostic resources.

84 Quantitative real-time polymerase chain reaction (qRT-PCR) is the gold-standard method for

85 clinical diagnosis, with high sensitivity and specificity, but these tests are accompanied by the need

86 for trained personnel, expensive reagents and instrumentation, and a significant amount of time to

87 execute. Facilities offering qRT-PCR sometimes require a week or longer to complete and return

88 the results to the patient. During this waiting period the undiagnosed individual may spread the

89 infection and/or receive delayed medical treatment. Moreover, due to the cost and relative

90 inaccessibility of qRT-PCR in both resource-limited and abundant settings, large-scale screening

91 using qRT-PCR at frequent intervals remains impractical to identify infected but asymptomatic or 
92 mildly symptomatic infections. Numerous studies have reported asymptomatic COVID-19 cases

93 as well as a variation in viral load within and between individuals at different time points,

94 suggesting the need for more frequent testing for informative surveillance.

95 Technologies alternate to qRT-PCR, such as rapid viral antigen detection, clustered 96 regularly interspaced short palindromic repeats (CRISPR), and loop-mediated isothermal 97 amplification (LAMP) of SARS-CoV-2 provide potential large-scale screening applications, yet 98 their implementation is stymied by requirements for qRT-PCR-like accuracy before they can reach 99 the market ${ }^{8}$. In countries such as India, where the qRT-PCR resources would not be sufficient to 100 cover monitoring of the population, the use of rapid antigen tests is well underway, ${ }^{9,10}$. In early 101 May 2020, the United States Food and Drug Administration (FDA) authorized the first antigen 102 test for the laboratory detection of COVID-19, citing a need for testing beyond molecular and 103 serological methods. Antigen testing detects the viral proteins rather than nucleic acids or human 104 antibodies, allowing for detection of an active infection with relative ease of sample collection and 105 assay. These rapid assays - like other commercially-available rapid antigen tests - can be mass106 produced at low prices and be administered by the average person without a laboratory or 107 instrumentation. These tests also take as little as 15 minutes to determine the result, enabling real108 time surveillance and/or diagnosis. Although antigen tests usually perform with high specificities 109 (true negative rate), their sensitivity (true positive rate) is often lower when compared to molecular 110 assays. While qRT-PCR can reach a limit of detection as low as $10^{2}$ genome copies per $\mathrm{mL}$, rapid 111 antigen testing detects viral protein that is assumed to correlate with approximately $10^{5}$ genome 112 copies per $\mathrm{mL}^{11}$.

113 We hypothesize that frequent antigen-based rapid testing even with lower sensitivities 114 compared to qRT-PCR - along with appropriate quarantine measures - can be more effective at 
115 decreasing COVID-19 spread than less frequent molecular testing of symptomatic individuals.

116 Keeping in mind the realities of daily testing in resource-limited regions, we also hypothesize that

117 testing frequency can be adjusted according to the prevalence of the disease; that is, an uptick in

118 reported cases should be accompanied by more frequent testing. During the viral incubation period,

119 high infectivity correlates with a high viral load that can be detected by either qRT-PCR or rapid

120 antigen testing ${ }^{12-16}$. Rapid tests thus optimize diagnosis for the most infectious individuals. Studies

121 also point to the relatively small window of time during an individual's incubation period in which

122 the qRT-PCR assay is more sensitive than rapid tests ${ }^{12}$.

123 In this study we report the clinical validation of two direct antigen rapid tests for detection

124 of SARS-CoV-2 spike glycoprotein (S) or nucleocapsid protein (N) using retrospectively collected

125 nasopharyngeal or nasal swab specimens. Using the clinical performance data, we develop a

126 modeling system to evaluate the impact of frequent rapid testing on COVID-19 spread and

127 outcomes using a variation of a SIR model, which has been previously used to model COVID-19

128 transmission ${ }^{17-23}$. We build on this model to incorporate quarantine states and testing protocols to

129 examine the effects of different testing regimes. This model distinguishes between undetected and

130 detected infections and separates severe cases, specifically, those requiring hospitalization, from

131 those less so, which is important for disease response systems such as intensive care unit triaging.

132 We simulate COVID-19 spread with rapid testing and model disease outcomes in three regions in 133 the United States and São José do Rio Preto, Brazil - the site of the clinical validation study - using

134 publicly available data. To date, COVID-19 modeling describes the course of disease spread in 135 response to social distancing and quarantine measures, and a previous simulation study has shown

136 that frequent testing with accuracies less than qRT-PCR, coupled with quarantine process and 137 social distancing, are predicted to significantly decrease infections $12,17,23-27$. This is the first 
138 modeling system using publicly-available data to simulate how potential public health strategies

139 based on testing performance, frequency, and geography impact the course of COVID-19 spread

140 and outcomes. Our findings suggest that a rapid test, even with sensitivities lower than molecular

141 tests, when strategically administered 2-3 times per week, will reduce COVID-19 spread,

142 hospitalizations, and deaths at a fraction of the cost of nucleic acid testing via qRT-PCR. Modern

143 surveillance systems should be well equipped with rapid testing tools to ensure that disease

144 tracking and control protocols are effective and well-tailored to national, regional, and community 145 needs.

147 RESULTS

148 Accuracy of Direct Antigen Rapid Tests Correlate with Viral Load Levels

149 Rapid antigen tests have recently been considered a viable source for first-line screening, 150 although concerns regarding the accuracy of these tests persist. We clinically validated two 151 different direct antigen rapid tests for the detection of either $\mathrm{N}$ or $\mathrm{S}$ from SARS-CoV-2 in 152 retrospectively collected nasal or nasopharyngeal swab specimens. Of the total number of nasal 153 swab specimens evaluated by qRT-PCR for amplification of SARS-CoV-2 N, S, and ORF1ab 154 genes, 100 tested positive and 90 tested negative (Table 1-2). The overall sensitivity and specificity 155 of the rapid antigen test for detection of SARS-CoV-2 N, evaluated across the nasal swab 156 specimens, was $80.0 \%$ and $91.1 \%$, respectively. Of the total number of nasopharyngeal swab 157 specimens evaluated by qRT-PCR for amplification of SARS-CoV-2 N, RNA-dependent RNA 158 polymerase (RdRp), and envelope (E) genes, 72 tested positive and 49 tested negative (Table 1, 159 Table 3). The overall sensitivity and specificity of the rapid antigen test for detection of SARS160 CoV-2 S, evaluated across the nasopharyngeal swab specimens was $84.7 \%$ and $85.7 \%$, 
161 respectively. Altogether, our data demonstrate that the sensitivity of the rapid antigen tests are 162 positively correlated to the viral load level (Table 1, Figure S1).

The $\mathrm{Ct}$ value indirectly quantifies the viral RNA copy number related to the viral load of

164 the sample for the specific assay ${ }^{28,29}$. Ct values represent the number of qRT-PCR cycles at which 165 generated fluorescence crosses a threshold during the linear amplification phase; $\mathrm{Ct}$ values are 166 therefore inversely related to the viral load. The sensitivity of both rapid antigen tests increases as $167 \mathrm{Ct}$ value decreases (Table 1). Moreover, because the $\mathrm{Ct}$ value is a variable unit based upon $\mathrm{qPCR}$ 168 protocol and instrumentation, we evaluated sensitivity against the percentile of positive cases 169 conditioned to $\mathrm{Ct}$ and found similar results. The sensitivity of the rapid antigen test for detection 170 of SARS-CoV-2 $\mathrm{N}$ increased from $80.0 \%$ at $\mathrm{Ct}$ values $<40$ to $95.8 \%$ at $\mathrm{Ct}$ values $<20$ 171 (Supplementary Fig. 1a). Similarly, the sensitivity of the rapid antigen test for detection of SARS$172 \mathrm{CoV}-2 \mathrm{~S}$ increased from $84.7 \%$ at $\mathrm{Ct}$ values $<35$ to $100.0 \%$ at $\mathrm{Ct}$ values $<15$ (Supplementary Fig. 173 1b). Taken together, the clinical data shows that the rapid antigen test performs with increasing 174 accuracy for individuals with a higher viral load, and potentially the most infectious ${ }^{13-16}$.

\section{An Enhanced Epidemiological SIDHRE-Q Model}

We propose an enhanced epidemiological modeling system, SIDHRE-Q, a variant of the

178 classical SIR model in order to expand our clinical validation study and to understand the effects 179 of using frequent rapid tests such as the rapid antigen test on COVID-19 outbreak dynamics. The 180 changes we make to the basic model to encompass the unique characteristics of the COVID-19 181 pandemic are similar to those presented by Giordano et al. (16) (Fig. 1, Supplementary Fig. 2). 182 The differential equations governing the evolution of the SIDHRE- $Q$ model and descriptions of 183 the parameter values are provided in the methods section (Equation 2, Table 4). 
An individual that begins in $\mathbf{S}$ may either transition to a Quarantine Uninfected $(\mathbf{Q}-\mathbf{U})$ state

185 via a false positive result or to an Infected Undetected (I) state via interaction with an infected

186 individual. Should an individual in $\mathbf{S}$ move into $\mathbf{Q}-\mathbf{U}$, they are quarantined for 14 days before

187 returning to $\mathbf{S}$, a time period chosen based on current knowledge of the infectious period of the

188 disease. One could also conceive of an effective strategy in which individuals exit quarantine after

189 producing a certain number of negative rapid tests in the days following their initial positive result 190 or confirm their negative result using qRT-PCR.

Given that those diagnosed are predominantly quarantined, individuals in I interact more

192 with the $\mathbf{S}$ population than do those in Infected Detected (D). Therefore, the infectious rate for $\mathbf{I}$ is

193 assumed to be significantly larger than for D. Furthermore, a region's ability to control an outbreak

194 is directly related to how quickly and effectively people in I test into $\mathbf{D}$, reducing their 195 infectiousness through quarantine. This study, in particular, highlights the critical role frequency 196 of testing, along with strict quarantine, has in mitigating the spread of the disease and provides 197 specific testing strategies based on rapid tests we predict to be highly effective.

In this model, we assume that individuals receive a positive diagnosis before developing

199 severe symptoms and that those with symptoms severe enough to be potentially fatal will go to the

200 hospital. If an individual develops symptoms, we assume they are tested daily until receiving a

201 positive result; hence, before severe symptoms develop, they will be diagnosed with high

202 probability. Those who do not develop symptoms are tested according to the frequency of tests

203 administered to the general population. Therefore, there is no modeled connection between I and

$204 \mathbf{H}$ or between I and E. Removing these assumptions would have negligible impact on the results 205 as these flows are very small. 
Should an individual test positive and transition to $\mathbf{D}$, they may either develop serious symptoms requiring care or recover. Those who develop serious symptoms and transition to state

$208 \mathbf{H}$ will then transition to either $\mathbf{R}$ or $\mathbf{E}$. The recovered population is inevitably tested, as infected 209 individuals may recover without being detected. Therefore, the Quarantined Recovered (Q-R) 210 state is introduced with the same connections to $\mathbf{R}$ as the connections between $\mathbf{S}$ and $\mathbf{Q}-\mathbf{U}$. Though 211 the reinfection rate of SARS-CoV-2 has been a point of recent debate, it is assumed that the number 212 of re-infected individuals is small ${ }^{30-34}$. Therefore, individuals cannot transition from $\mathbf{R}$ to $\mathbf{S}$, hence 213 the separately categorized quarantined populations.

214 We considered several variations and extensions of the SIDHRE-Q model. In simulations, 215 we tested additional states, such as those in the SIDARTHE model, which include distinctions 216 between symptomatic and asymptomatic cases for both detected and undetected populations ${ }^{17}$. 217 Incorporating information about the correlations between viral load and infectivity and sensitivity 218 were also considered. Altogether, our modeling system has been well tuned to predict the impact 219 of high frequency rapid testing on COVID-19 spread and outcomes.

220

221 Frequent Rapid Testing with Actionable Quarantining Dramatically Reduces Disease 222 Spread

223 In order to demonstrate how strategies could affect the disease spread in different 224 geographies and demographics, we used surveillance data obtained from regions of varying 225 characteristics: the state of Massachusetts (MA), New York City (NYC), Los Angeles (LA), and 226 São José do Rio Preto (SJRP), Brazil, the site of the rapid antigen test clinical validation study.

227 These regions are also selected in our study due to the readily available surveillance data provided 228 by the local governments. We fit the model to the data from each region starting 1 April 2020. At 
229 this time point the disease reportedly is most advanced in NYC and least advanced in SJRP, Brazil

230 with estimated cumulative infection rates of $7.11 \%$ and $0.12 \%$, respectively.

231 After calibrating the SIDHRE-Q model, the disease spread is observed with varying

232 validated rapid antigen test performances and frequencies (Fig. 2). Sensitivity (the ratio of true

233 positives to the total number of positives) and specificity (the ratio of true negatives to the total

234 number of negatives) compared to gold-standard qRT-PCR were used as measures of test 235 accuracy.

236 The rapid test frequency is varied while maintaining an accuracy of $80 \%$ sensitivity and

$23790 \%$ specificity, comparable to our clinical data collected in SJRP, Brazil. These testing scenarios

238 are then compared to symptomatic testing, in which individuals receive a rapid test only when

239 presenting symptoms, via either a rapid test or qRT-PCR. Since the primary testing regiment

240 deployed in MA, LA, NYC and SJRP, Brazil is qRT-PCR-based and focused on symptomatic

241 individuals, the symptomatic testing protocol via qRT-PCR is directly estimated from the data to

242 be the rate $v$ (Table 4).

243 The difference between the qRT-PCR and rapid test simulations (red and orange lines,

244 respectively) is therefore only sensitivity of testing (Fig. 2). We assumed that test outcome

245 probability is a function only of whether an individual is infected and independent of other factors;

246 one can consider this a lower bound on effectiveness of a strategy, as sensitivity and infectivity

247 are often positively correlated with antigen testing.

248 To better understand the effect of rapid testing frequency and performance on healthcare

249 capacity and mortality rates, we simulate the testing strategy with $30 \%-90 \%$ sensitivity each with

$25080 \%$ or $90 \%$ specificity against the symptomatic testing strategy (Supplementary Fig. 3). 
As per our hypothesis, frequency and symptom-based testing dramatically reduced

252 infections, simultaneous hospitalizations, and total deaths when compared to the purely symptom-

253 based testing regiments, and infections, hospitalization, and death were reduced as frequency

254 increased. Although testing every day was clearly most effective, even testing every fourteen days

255 with an imperfect test gave an improvement over symptomatic testing with qRT-PCR. While the

256 strategy works best when implemented at the very beginning of an outbreak, as demonstrated by

257 the results in SJRP, Brazil, it also works to curb an outbreak that is already large, as demonstrated

258 by the results in NYC. The difference between frequencies is more noticeable when the testing

259 strategy is applied to the outbreak in NYC, leading us to hypothesize that smaller outbreaks require

260 a lower testing frequency than larger ones; note the difference between the dependence on

261 frequency to curb a small initial outbreak in SJRP, Brazil versus a large one in NYC (Fig. 3).

262 For test performance of $80 \%$ sensitivity and $90 \%$ specificity, the percent of the population

263 that has been infected in total from the beginning of the outbreak to mid-July drops from $18 \%$

264 (MA), 11\% (LA), 26\% (NYC), and 11\% (SJRP, Brazil) to 3\%, 2\%, 12\%, and 0.26\%, respectively,

265 using a weekly rapid testing and quarantine strategy (with regards to predictions of overall

266 infection rates, other studies based on seroprevalence and epidemiological predictions have

267 reached similar conclusions ${ }^{35,36}$ ). If testing is increased to once every three days, these numbers

268 drop further to 1.6\% (MA), 1.4\% (LA), 9.5\% (NYC), and 0.19\% (SJRP, Brazil) (Table S1).

To further examine the relationship between frequency and sensitivity, we modeled the

270 maximum number of individuals in a given state over the 105-day time period for four geographic

271 regions (Fig. 3, Supplementary Fig. 4). In all four geographic regions, as frequency of testing

272 increases, the total infections, maximum simultaneous hospitalizations, and total deaths converge

273 to small percentages regardless of the sensitivity at high frequencies. It is clear that the difference 
274 in frequency required to achieve the same result using tests of differing sensitivities is very small.

275 For example, we predict that for the outbreak in LA, a testing strategy started on 1 April of every

27610 days using a test of sensitivity $90 \%$ would have resulted in $2.5 \%$ of the population having been

277 infected, while using a test of sensitivity $30 \%$ would require a strategy of every 5 days to achieve

278 the same number. Thus, we conclude that frequency is more important than sensitivity in curbing

279 the spread, and a large range of sensitivities prove effective when testing sufficiently often

280 (Supplementary Fig. 4). How frequently, exactly, depends on the specific outbreak and what stage

281 it is in, which leads us to the location-based deployment strategy discussed in a later section.

282 Frequency of testing can be significantly reduced to effectively contain the disease once the initial

283 outbreak has been controlled; it is clear that this takes only a matter of weeks (Fig. 2).

284 On the other hand, according to the specificity of the rapid test and the quarantine duration, 285 larger testing frequency result in a larger percent of the population quarantined (Fig. 2). Assuming 286 a 90\% rapid test specificity and 14-day quarantine duration, for the 1-, 3- and 7-day frequencies 287 almost $60 \%, 38 \%$ and $20 \%$ of the population, respectively, would be quarantined. This figure may 288 be reduced with additional rules for exiting quarantine early, such as after complementary testing. 289 An example of such a strategy is that individuals who test positive are required to either quarantine 290 for two weeks or produce two consecutive negative rapid tests in the two days following their 291 positive result. Assuming $80 \%$ sensitivity and $90 \%$ specificity, those individuals will reenter the 292 public while still infected with probability 0.04 . If uninfected, that individual will exit quarantine 293 after two days with probability 0.81 . However, a compromise between the reduction of infections 294 and the proportion of the population in quarantine would be part of the planning for the appropriate 295 testing protocol in each community or region. 
Additionally, while high frequency may be necessary to contain a large outbreak initially,

297 relatively infrequent testing, such as every one or two weeks, is sufficient to keep controlled

298 outbreaks small, while reducing the number of quarantined individuals to less than $10 \%$ of the 299 population using a two-week mandatory quarantine.

A County-Based Testing Strategy Offers a Cost-effective Approach to Large-scale COVID-

\section{Surveillance}

To examine the effects of resource-strategic testing schemes, we modeled the COVID-19

304 prevalence by varying testing frequency across counties of California. For this analysis, only

305 California was analyzed because of the accessibility of the county level data and the variability of 306 spread dynamics of the outbreaks between counties. In this scheme, the percent of active infected 307 detected individuals in a county determines the frequency of testing. We define thresholds for the 308 number of active detected infections that, when hit, initiate testing protocols of different 309 frequencies depending on the threshold hit. We first tested evenly spaced thresholds for the number 310 of detected active infections up to $1 \%$ of the population, but later adopted thresholds that were 311 determined according to Equation 1. In Equation 1, D= population of state $\mathbf{D}$ at the time of testing. $312 T=$ number of active infections which, if reached, initiates everyday testing. The days between 313 tests are rounded to the closest integer value.

316 The days between tests are chosen such that the detected active infections should remain near to

317 or below $T$. If the initial detected active infections are greater than $T$, then the testing frequency 318 of 1 will cause infections to rapidly drop. Both the threshold at which everyday testing begins and 
319 the coefficient of $\log _{2} T / D$ can be modified to produce a strategy that is more or less frequent in 320 testing or resource effective; a range of days between tests from 14 days to 1 day are used (Fig. 321 4a). The purpose of this strategy is to tailor testing based on the specific characteristics of local 322 outbreaks in order to mitigate the overall spread faster and more efficiently. A scan over different 323 choices of $T$ is shown in Fig. 4 b; the threshold we choose in Fig. $4 \mathrm{a}$ is $0.05 \%$ because it is successful 324 in curbing the outbreak within the time period we consider. While the choices work for the 325 epidemic in California at the point we start our simulations, 10 April, they do not necessarily reflect 326 the most resource effective choices everywhere. Our analysis could be redone to select the best 327 fine-grained strategy in other states or metropolitan areas. Using a rapid test with a sensitivity of $80 \%$ and specificity of $90 \%$, the county-based testing 329 with threshold $0.05 \%$ reduces the active infections from $0.94 \%$ to $0.0005 \%$, while the uniform 330 strategy with tests administered every 7 days results in double the number of active infections (Fig. 331 4a). As the threshold is reduced, the total cost increases while the cumulative infections, maximum 332 percentage hospitalized, and cumulative deaths all decrease (Fig. 4b). Appropriate choice of 333 threshold is dependent on the severity of outbreaks in a specific region and available resources, 334 both logistically and fiscally. With regional data, such as that from California used to produce Fig. $3354 \mathrm{~b}$, this study can be reproduced to calculate an efficient testing strategy that will effectively curb 336 outbreaks of differing severities in any geographic entity.

337 Strategy B in Fig. 4 consists of qRT-PCR testing uniformly applied to the highlighted 338 population with a frequency of once weekly. The average cost per person per day is just under $\$ 15$. 339 Despite this frequency and the accuracy of qRT-PCR, the strategy does not succeed in curbing the 340 spread as fast as strategy A, which uses a testing sensitivity and specificity of $80 \%$ and $90 \%$, 341 respectively, and testing frequency that vary between counties depending on the proportion of their 
342 population that is currently infected. The total cost for strategy $\mathrm{A}$ is estimated at a fraction of the

343 other at $\$ 1.53$ per person per day.

344

\section{DISCUSSION}

346 In this study we examine the potential effects of a novel testing strategy to limit the spread

347 of SARS-CoV-2 utilizing rapid antigen test screening approaches. Our clinical data and SIDHRE-

$348 Q$ modeling system demonstrate that 1) frequent rapid testing even at a range of accuracies is 349 effective at reducing COVID-19 spread, 2) rapid antigen tests are a viable source for this strategy

350 and diagnose the most infectious individuals, and 3) strategic geographic-based testing can

351 optimize disease control with the amount of available resources. The public has witnessed and

352 experienced symptomatic individuals being denied testing due to shortages, and few testing

353 structures for asymptomatic or mildly symptomatic individuals - a significant source of disease

354 spread. Though several factors contributed to the stymied early response measures, such as

355 lockdown and quarantine protocols and adherence, severe testing bottlenecks are a significant

356 culprit ${ }^{37-39}$. Early control measures have been shown to decrease lives lost by several orders of 357 magnitude ${ }^{40}$. These challenges, though exacerbated during the early months of the pandemic, 358 remain at the forefront of the public health crises.

359 Diagnosis of SARS-CoV-2 infection by qRT-PCR is the current standard of care, yet 360 remains expensive and requires a laboratory and experienced personnel for sample preparations 361 and experimentation. The turnaround time for results can be up to 10 days, preventing people from 362 either leaving quarantine if they are negative, or delaying critical care and infecting others if they 363 are positive ${ }^{41}$. This current testing scheme moreover yields incomplete surveillance data on which 364 response efforts such as societal reopening and hospital management depend. Though qRT-PCR 
365 is considered the gold-standard diagnostic method because of its high sensitivity and specificity,

366 the logistical hurdles render it unrealistic for large-scale screening.

As qRT-PCR remains impractical for this strategy, and rapid tests are facing regulatory

368 challenges because they do not perform with qRT-PCR-like accuracy, rapid test screening is either

369 nonexistent in several countries or symptom-based. Even under best-case assumptions, findings

370 have shown that symptom and risk-based screening strategies miss more than half of the infected

371 individuals ${ }^{42}$. Some have argued that the need for widespread testing is overstated due to the

372 variability in test sensitivity and specificity ${ }^{43}$. Here, we present alternative large-scale diagnostic

373 tools to qRT-PCR, and show that test performance, though valuable, is secondary to widespread

374 test frequency, which is enabled by accessibility and turnaround time. Furthermore, test

375 affordability is essential for the successful implementation in communities most affected by

376 infection and will to speed up the safe opening and functioning of the viral sectors of the economy.

377 Giordano et al. has modeled the evolution of SARS-CoV-2 spread, introducing a diagnosed

378 state to elucidate the importance of population-wide testing ${ }^{17}$. Mina et al. has examined how

379 various test sensitivities and frequencies affect the reproductive number ${ }^{12}$. We build upon these

380 findings to show how in affected United States and Brazil regions, population-wide frequent and

381 rapid testing schemes, with sensitivities ranging from $30 \%-90 \%$, can be more effective in curbing

382 the pandemic than a PCR-based scheme. Integrating real-world surveillance and clinical data into

383 our modeling system has allowed us to incorporate regional differences - such as variances in

384 healthcare access, state health policy and adherence, state GDP, and environmental factors - under

385 the same model. Significantly, our findings hold true across Massachusetts, New York City, Los

386 Angeles, and São José do Rio Preto, Brazil. We also present the economic considerations of these

387 testing regimes, showing that widespread rapid testing is more cost efficient than less frequent 
qRT-PCR testing. In line with these economic considerations, our model demonstrates the 389 effectiveness of a geographic-based frequent testing regime, in which high disease prevalence areas receive more frequent testing than low disease prevalence areas.

Since COVID-19 is known to affect certain demographics differently, modeling would

392 benefit from incorporating demographic information correlated with disease progression and 393 spread to define sub-models and sets of parameters accordingly. Age, pre-existing conditions, job 394 types, and density of population are examples of possible categories, each of which influence the 395 risk of contracting and/or dying from COVID-19. Further studies would benefit from incorporating 396 these ideas to better understand the effectiveness of rapid testing on identifying potential super 397 spreading events. Future public health prevention programs should use the proposed modeling 398 system to develop and test scenarios for precision testing and prevention.

400 as a rapid antigen-based test for the detection of SARS-CoV-2 proteins. We show that the rapid 401 antigen tests perform with a range of accuracies under which disease spread can be dramatically 402 mitigated under our model. Notably, the sensitivity is correlated to the individual's viral load, 403 effectively diagnosing those who are potentially the most infectious with the highest accuracy. Our 404 findings are significant because rapid antigen tests are cheaper than qRT-PCR, can be mass 405 produced to millions per day, present results within 15 minutes, and can be administered by a 406 nonexpert without a lab or special equipment.

407 There are several policy implications for these findings. First, our model supports that 408 systems of high frequency rapid testing should be implemented as a first-line screening method. 409 This can be first enabled by a more holistic regulatory evaluation of rapid diagnostics, such that 410 policy emphasizes accessibility and turnaround time even under a range of accuracies. One can 
411 imagine a less accurate, though rapid method of first-line screening in schools, public

412 transportation, and airports, or even at home, and a qRT-PCR-based method for second-line

413 screening (testing those who present severe symptoms or have been in contact with infected

414 individuals, testing in a clinical setting, etc). Second, our cost analysis and rapid antigen test data

415 present a viable and potentially more cost-effective method for screening. Third, our county-based

416 testing scheme presents a possible method for wide-scale screening while optimizing resources.

417 Future studies should investigate how this selective testing strategy can be applied to different

418 location scales to further inform health policy. Moreover, though our models analyze regions in

419 the United States and Brazil, similar testing strategies can be considered globally in both resource

420 limited and abundant settings due to the higher accessibility of rapid tests compared to qRT-PCR.

421 We emphasize that integral to the effectiveness of diagnostic schemes is 1) the proper

422 adherence to quarantine measures and 2) the combined use of a variety of diagnostic methods

423 including nucleic acid, antigen, and antibody tests. According to these models, rapid antigen tests

424 are an ideal tool for first-line screening. Clinical molecular tests such as qRT-PCR are vital to the

425 diagnostic landscape, particularly to re-test suspected cases that were negative on the rapid test.

426 Because rapid tests present a higher rate of false negatives, methods such as qRT-PCR remain

427 integral to second-line screening. Antibody tests provide important information for immunity and

428 vaccination purposes as well as epidemiological surveillance. This model also assumes that 429 individuals will quarantine themselves before being tested and for 14 days following a positive 430 diagnostic result.

431 Our simulations combined with real-world data demonstrate a robust modeling system and

432 elucidates the significance of this novel testing strategy. However, there are important limitations

433 to be considered. Differences in disease reporting between the geographical regions and the 
434 incomplete nature of COVID-19 surveillance data, often due to the lack of testing, are not 435 considered in the model. It is imperative that the testing results, hospitalization and death statistics, 436 and changes in protocol are reported in real-time to scientists and policy makers so that models

437 can be accurately tuned as the pandemic develops. The model also does not take into account 438 infrastructural limitations such as hospital capacity. Though the rapid antigen test offers several 439 advantages such as affordability, fast turnaround time, and ease of mass production, we are also 440 assuming that there are systems in place to implement frequent and safe low-cost screening across 441 different communities and settings.

442 Our model underscores the need for a point-of-care or at-home test for frequent screening,

443 particularly as lockdown restrictions ease. Regulatory agencies can work towards evaluating rapid 444 tests to alternative standards other than comparison to high sensitivity molecular diagnostics, as 445 our model shows that frequency and scale of testing may overcome lower sensitivities. Rather, we 446 could refocus policy to implement first-line screening that optimizes accuracy with efficiency and 447 equitability.

\section{METHODS}

\section{Development of Direct Antigen Rapid Tests for the Detection of SARS-CoV-2}

454 We developed a direct antigen rapid test for the detection of the nucleocapsid protein or 455 spike glycoprotein from SARS-CoV-2 in nasal or nasopharyngeal swab specimens as previously 456 described ${ }^{44}$. Briefly, the rapid antigen tests are immunochromatographic format with a visual 
457 readout using anti-N or anti-S mouse monoclonal antibodies (E25Bio, Inc., Cambridge, MA, USA)

458 that are either coupled to $40 \mathrm{~nm}$ gold nanoparticles (Abcam, Cambridge, UK) or adsorbed to 459 nitrocellulose membranes (Sartorius, Goettingen, Germany). Each rapid antigen test has a control 460 area adjacent to the paper absorbent pad; the control is an anti-mouse Fc domain antibody (Leinco 461 Technologies, Fenton, MO, USA) that will capture any of the antibody-conjugated gold 462 nanoparticles to generate a control visual signal. A visual signal at the test area reflects SARS$463 \mathrm{CoV}-2 \mathrm{~N}$ or $\mathrm{S}$ that is "sandwiched" between an anti-N or anti-S antibody adsorbed to the 464 nitrocellulose membrane and a second anti-N or anti-S antibody covalently coupled to visible gold 465 nanoparticles.

467 Validation of Direct Antigen Rapid Test for the Detection of SARS-CoV-2

468 In a retrospective study of nasal swab specimens form human patients, we compared the 469 accuracy of the rapid antigen test for detection of SARS-CoV-2 N to the viral loads of individuals. 470 Nasal swab specimens $(\mathrm{n}=190)$ were tested following approved human subjects use protocols. The 471 nasal swab specimens were banked frozen from suspected patients submitted to PATH for routine

472 COVID diagnosis. Prior to using the rapid test, the nasal swab specimens were validated by qRT473 PCR using the FDA EUA ThermoFisher/AppliedBiosystems TaqPATH COVID-19 Combo Kit

474 (ThermoFisher, Waltham, MA USA). The primary study under which the samples and data were 475 collected received ethical clearance from the PATH Research Ethics Committee, protocol number 476 00004244. The nasal swab specimens were de-identified, containing no demographic data, prior 477 to analysis.

478 The nasal swabs were originally collected in $1 \mathrm{~mL}$ PBS, where $50 \mu 1$ was mixed with $50 \mu \mathrm{l}$ 479 of Solution Buffer $(0.9 \% \mathrm{NaCl}$ and $0.1 \%$ Triton $\mathrm{X}-100)$. The $100 \mu \mathrm{l}$ mixture was then pipetted 
onto the rapid antigen test for SARS-CoV-2 nucleocapsid detection and allowed to react for 15

481 minutes. After processing of the rapid antigen test, the visual positive or negative signal was 482 documented.

483 Additionally, in a retrospective study of nasopharyngeal swab specimens from human 484 patients, we compared the accuracy of the rapid antigen test to the viral load of individuals. 485 Nasopharyngeal swab specimens $(n=121)$ were tested in Brazil following approved human 486 subjects use protocols. The age of study participants ranged from 1 to 95 years with an overall 487 median of 37 years (interquartile range, 27-51 years), and 62\% were female. The demographic 488 summary of the patients are included in Table S2. The nasopharyngeal swab specimens were 489 banked refrigerated or frozen samples from suspected patients submitted to the lab for routine 490 COVID diagnosis. Prior to using the rapid test, the nasopharyngeal swab samples were validated 491 by qRT-PCR using GeneFinder ${ }^{\mathrm{TM}}$ COVID-19 Plus RealAmp Kit (OSANGHealtcare, Anyang-si, 492 Gyeonggi-do, Republic of Korea I). The primary study under which the samples and data were 493 collected received ethical clearance from the Faculdade de Medicina de São José do Rio Preto 494 (FAMERP), protocol number 31588920.0.0000.5415. All excess samples and corresponding data 495 were banked and de-identified prior to the analyses. Nasopharyngeal swab specimens $(1 \mathrm{~mL})$ were concentrated using Vivaspin 500 centrifugal 497 concentrator (Sartorius, Goettingen, Germany) at 12,000 x g for 10 minutes. The concentrated 498 nasopharyngeal swab specimen retentate was transferred to a collection tube and the rapid antigen 499 test for SARS-CoV-2 spike detection was inserted into the tube with the retentate and allowed to 500 react for 15 minutes. After processing of the rapid antigen test, the visual positive or negative 501 signal was documented. 


\section{Data for Modeling}

504

As of August 2020, the United States and Brazil have the highest number of confirmed

505 COVID-19 cases and deaths worldwide, with both countries reporting their first case on 26

506 February 202) ${ }^{1}$. Although several affected US regions could have been modeled, we look at data

507 from Massachusetts, New York, and Los Angeles: these regions each contained "hotspots", or 508 areas of surging COVID-19 cases, at different points in time during the pandemic and have 509 publicly available government-provided surveillance data. Our model is fit using data over 105

510 days beginning on April 1 for Fig. 2 and Fig. 3, and 105 days beginning on April 10 for Fig. 4 (see

511 "Modeling Parameters" in Methods). In order to understand the various testing proposals on a

512 global scale, we performed our clinical study in and expanded the modeling study to Brazil. The

513 specific data we use to fit our model are cumulative confirmed cases, total deaths, and number of 514 daily hospitalizations due to COVID-19. This surveillance data was retrieved from government515 provided online databases ${ }^{45-51}$.

516

\section{Modeling Parameters}

518 Equation 2 below provides the exact differential equations governing the model.

$\begin{array}{rll}\mathrm{d} \mathbf{S}= & -\mathbf{S}(\alpha \mathbf{I}+\eta \mathbf{D}+\gamma) & +\psi \mathbf{Q}_{\mathbf{U}} \\ \mathrm{d} \mathbf{I}= & -\mathbf{I}(\varepsilon+\lambda+\nu) & +\mathbf{S}(\alpha \mathbf{I}+\eta \mathbf{D}) \\ \mathrm{d} \mathbf{D}= & -\mathbf{D}\left(\frac{\mathbf{D}+\mathbf{I}}{\mathbf{D}} \mu+\rho\right) & +\mathbf{I}(\nu+\varepsilon) \\ \mathrm{d} \mathbf{H}= & -\mathbf{H}(\sigma+\tau) & +\mu(\mathbf{D}+\mathbf{I}) \\ \mathrm{d} \mathbf{E}= & & +\tau \mathbf{H} \\ \mathrm{d} \mathbf{R}= & -\gamma \mathbf{R} & +\rho \mathbf{D}+\lambda \mathbf{I}+\sigma \mathbf{H}+\psi \mathbf{Q}_{\mathbf{R}} \\ \mathrm{d} \mathbf{Q}_{\mathbf{U}}= & -\psi \mathbf{Q}_{\mathbf{U}} & +\gamma \mathbf{S} \\ \mathrm{d} \mathbf{Q}_{\mathbf{R}}= & -\psi \mathbf{Q}_{\mathbf{R}} & +\gamma \mathbf{R}\end{array}$


520 In order to determine the values of the parameters defining the flows between states, we use a least

521 squares regression performed at seven day intervals in the datasets to which we fit. This allows

522 the model to take into account the time dependent nature of the parameters, which rely on factors

523 such as social distancing regulations and changes in testing capacity. We also fit window sizes

524 between 1 and 21 days and find that while the fit degrades with larger window size, the overall

525 shape of the fits do not change. We choose seven days assuming policy changes take a week to

526 become effective and that reasonable parameters can be expected to change within this time period.

527 Also, the seven day window size accounts for the fact that often data is not reported as diligently

528 over the weekend. Time series of the values of the parameters for the geographic locations

529 discussed in this paper are included in Supplementary Fig. 5.

530 Given the restrictions on data available for the populations of various states, varying all of

531 the parameters results in an over parameterized system. Therefore, a subset of the model

532 parameters are fit while the others are either extracted from other sources; see Table 4 . The fitting

533 procedure minimizes the sum of the squared residuals of the total cases, current daily

534 hospitalizations, cumulative deaths, and percentage of total infected individuals currently

535 hospitalized. The first three are present in the data sets while the latter is derived from the estimates

536 of the ratio between infected undetected to infected detected individuals from the CDC Laboratory

537 Seroprevalence Survey Data ${ }^{52}$. While this ratio changes over time, the percentage of infected

538 individuals developing severe symptoms should remain roughly constant throughout the course of

539 the epidemic in the different locations studied.

540 We consider the data sets for outbreaks in MA, NYC, LA, and SJRP, Brazil ${ }^{45-50}$. While

541 each location has testing and fatality information dating back to January, hospitalization data was

542 not included until late March (for NYC and SJRP) and April (for MA and LA). Hence we begin 
543 our fitting procedure and testing strategy on 1 April for each of the data sets; by this point, the

544 outbreak is advanced in NYC, substantial in MA, non-negligible, but far from its peak, in LA, and

545 in early stages in SJRP, Brazil. Starting simulations at various stages of the outbreak allows one

546 to see the difference in results between when a testing strategy is administered.

547 In order to determine the effectiveness of the county-based strategy when applied to the

548 state of California, we also fit all of the counties in California with a population greater than $1.5 \%$

549 of that of the entire state and with greater than zero deaths. The results do not depend on these

550 selections, but instead suggest a practical criteria to administer limited resources. The fitting is

551 done starting 10 April for these counties, as at this point the outbreak is sufficiently well-

552 documented in each to successfully model. For the county-level data we compute a seven day

553 running average of each of the data sets to which we then fit in order to smooth out fluctuations in

554 the data, likely due to reporting, which are more significant here than in the other data sets

555 considered, as the county populations are smaller and hence discrepancies impact the smoothness

556 of the data more. The fits for each of the counties can be found in Supplementary Fig. 6.

557 As one can see from Fig. 1, these data sets are particularly not smooth, which indicates

558 inefficiencies in reporting. Additionally, it is difficult to gauge their consistency within the dates

559 provided or to compare between locations, as reporting mechanisms changed over time within the

560 same locations. Despite this lack of consistency, our model and fitting mechanism was successful

561 in reproducing the progress of the outbreak in each data set studied.

562

563 DATA AVAILABILITY

564 The authors confirm that the data supporting the findings of this study are available within the 565 article and/or its supplementary materials; any other data will be made available upon request. 
CODE AVAILABILITY

568 Full code can be found on github: https://github.com/badeaa3/COVID19 Rapid Testing. The 569 code is written using python with the packages scipy, numpy, lmfit, matplotlib and plotly ${ }^{53-57}$. 570

571 SUPPLEMENTARY MATERIALS

572

573 Supplementary Table 1. Summary of results of COVID-19 outcomes in 3 US Regions and 574 Brazil as a result of Frequent Rapid Testing Protocol using SIDHRE-Q Model. 575

576 Supplementary Table 2. Demographic and clinical summary of patients evaluated by the 577 SARS-CoV-2 Direct Antigen Rapid Test (DART).

578

579 Supplementary Fig. 1. Performance of direct antigen rapid test (DART) for the detection of 580 SARS-CoV-2 (A) nuceleocapsid protein and (B) spike glycoprotein. Shown are the percentile 581 positive cases of the total positive population conditioned to qRT-PCR Cycle Threshold (Ct).

582 Percentile Positive ranks the samples in order of high $\mathrm{Ct}$ to low $\mathrm{Ct}$. DART sensitivity is

583 determined by calculating true positive agreement to qRT-PCR; the plot uses an $\mathrm{ax}^{\mathrm{b}}+\mathrm{c}$ fit and $58495 \%$ confidence intervals for the sensitivity.

586 Supplementary Fig. 2. Graphical scheme displaying the relationships between the stages of 587 quarantine and infection in SIDHRE-Q model: Q-U, quarantine uninfected; S, susceptible 588 (uninfected); I, infected undetected (pre-testing and infected); D, infected detected (infection 
589 diagnosis through testing); $\mathbf{H}$, hospitalized (infected with life threatening symptom progression);

$590 \mathbf{R}$, recovered (healed); E, extinct (dead); and Q-R, quarantine recovered (healed but in

591 quarantine by false positive testing).

Supplementary Fig. 3. COVID-19 Outcomes as a result of Frequent Rapid Testing Protocol

594 with variable test performances using SIDHRE- $Q$ Model. The Cumulative Detected Infected,

595 Hospitalized, Deceased, Active Infections, Recovered, and Quarantined are modeled over 105

596 days (top to bottom) using reported data from 4 global regions: Massachusetts, Los Angeles,

597 New York City, and São José do Rio Preto in Brazil (left to right). The COVID-19 population

598 spread and outcomes are modeled under a Rapid Testing Protocol with variable testing

599 frequencies ranging from 1-21 days between tests, and variable test performances: $90 \%$

600 specificity with $90 \%$ sensitivity (A), 70\% sensitivity (B), 50\% sensitivity (C), and 30\%

601 sensitivity (D); and 80\% specificity with 90\% sensitivity (E), 70\% sensitivity (F), 50\%

602 sensitivity $(\mathrm{G})$, and $30 \%$ sensitivity $(\mathrm{H})$. This protocol is compared to a symptom-based Rapid

603 Testing protocol and a symptom-based qRT-PCR protocol.

604

605 Supplementary Fig. 4. Effect of Rapid Testing Protocol under variable testing sensitivities

606 and increasing frequency under the SIDHRE-Q Model. The Cumulative Infections,

607 Maximum Simultaneously Hospitalized, and Deceased populations are modeled for

608 Massachusetts, Los Angeles, New York City, and São José do Rio Preto in Brazil. The effect of

609 increasing frequency of testing is modeled for various testing sensitivities (30\%-90\%) with an

$61080 \%$ specificity. 
613 Supplementary Fig. 5. Time series of the four fitted parameters $\alpha, v, \mu$, and $\tau$ (left to right)

614 for MA, LA, NYC, and SJRP (top to bottom). See Table 4 in the Methods section for an

615 explanation of the parameters. The values are extracted every seven days from data provided by

616 the respective regions. The parameters vary significantly over time and location. Flat points

617 occur during the seven day windows where the parameters are held constant. The fitting

618 procedure is also outlined in the Methods section.

619

620 Supplementary Fig. 6. Time series of the three fitted pieces of data Cumulative Cases, Daily

621 Hospitalized, and Cumulative Deaths (left to right) for each county receiving testing in CA;

622 Ventura (2A), Stanislaus (2B), Santa Clara (2C), San Joaquin (2D), San Francisco (2E), San

623 Diego (2F), San Bernardino (2G), Sacramento (2H), Orange (2I), Los Angeles (2J), Kern (2K),

624 Fresno (2L), Alameda (2M). The counties included satisfy two requirements: population greater

625 than $1.5 \%$ of the total CA population and nonzero total number of deaths at each point in time.

626 The fitting procedure is outlined in the Methods section.

627

628

629 References

630 1. Coronavirus Disease (COVID-19) Situation Reports. https:/www.who.int/emergencies/diseases/novel-

$631 \quad$ coronavirus-2019/situation-reports.

632 2. Menkir, T. F. et al. Estimating the number of undetected COVID-19 cases exported internationally from all of 633 China. medRxiv (2020) doi:10.1101/2020.03.23.20038331.

634 3. Ivorra, B., Ferrández, M. R., Vela-Pérez, M. \& Ramos, A. M. Mathematical modeling of the spread of the 635 coronavirus disease 2019 (COVID-19) taking into account the undetected infections. The case of China.

636 Commun Nonlinear Sci Numer Simul 105303 (2020) doi:10.1016/j.cnsns.2020.105303. 
637 4. Salathé, M. et al. COVID-19 epidemic in Switzerland: on the importance of testing, contact tracing and 638 isolation. Swiss Med Wkly 150, w20225 (2020).

639 5. Lau, H. et al. Evaluating the massive underreporting and undertesting of COVID-19 cases in multiple global $640 \quad$ epicenters. Pulmonology (2020) doi:10.1016/j.pulmoe.2020.05.015.

641 6. Silverman, J. D., Hupert, N. \& Washburne, A. D. Using influenza surveillance networks to estimate state-

642 specific prevalence of SARS-CoV-2 in the United States. Science Translational Medicine (2020)

643 doi:10.1126/scitranslmed.abc1126.

644 7. Böhning, D., Rocchetti, I., Maruotti, A. \& Holling, H. Estimating the undetected infections in the Covid-19

645 outbreak by harnessing capture-recapture methods. Int. J. Infect. Dis. 97, 197-201 (2020).

646 8. Baek, Y. H. et al. Development of a reverse transcription-loop-mediated isothermal amplification as a rapid 647 early-detection method for novel SARS-CoV-2. Emerg Microbes Infect 9, 998-1007 (2020).

648 9. Leo, L. Mylab gets commercial approval from ICMR for Covid-19 antigen rapid testing kit. Livemint 649 https://www.livemint.com/news/india/mylab-gets-commercial-approval-from-icmr-for-covid-19-antigen-rapid650 testing-kit-11595434040321.html (2020).

651 10. Dey, S. Coronavirus testing: Rapid antigen tests now make up nearly half of daily checks | India News - Times 652 of India. The Times of India https://timesofindia.indiatimes.com/india/rapid-antigen-tests-now-make-up-nearly653 half-of-daily-checks/articleshow/77340459.cms.

654 11. Vogels, C. B. F. et al. Analytical sensitivity and efficiency comparisons of SARS-CoV-2 RT-qPCR primer655 probe sets. Nat Microbiol (2020) doi:10.1038/s41564-020-0761-6.

656 12. Larremore, D. B. et al. Test sensitivity is secondary to frequency and turnaround time for COVID-19 657 surveillance. medRxiv (2020) doi:10.1101/2020.06.22.20136309.

658 13. Shen, Z. et al. Superspreading SARS events, Beijing, 2003. Emerging Infect. Dis. 10, 256-260 (2004).

659 14. Peiris, J. S. M. et al. Clinical progression and viral load in a community outbreak of coronavirus-associated 660 SARS pneumonia: a prospective study. Lancet 361, 1767-1772 (2003).

661 15. He, X. et al. Temporal dynamics in viral shedding and transmissibility of COVID-19. Nat. Med. 26, 672-675 $662 \quad(2020)$

663 16. Bullard, J. et al. Predicting infectious SARS-CoV-2 from diagnostic samples. Clin. Infect. Dis. (2020) 664 doi:10.1093/cid/ciaa638. 
17. Giordano, G. et al. Modelling the COVID-19 epidemic and implementation of population-wide interventions in Italy. Nat. Med. 26, 855-860 (2020).

667 18. Choi, S. \& Ki, M. Estimating the reproductive number and the outbreak size of COVID-19 in Korea. Epidemiol $668 \quad$ Health 42, e2020011 (2020).

669 19. Wei, Y. Y. et al. [Fitting and forecasting the trend of COVID-19 by SEIR(+CAQ) dynamic model]. Zhonghua 670 Liu Xing Bing Xue Za Zhi 41, 470-475 (2020).

671 20. Yang, Z. et al. Modified SEIR and AI prediction of the epidemics trend of COVID-19 in China under public 672 health interventions. J Thorac Dis 12, 165-174 (2020).

673 21. Cao, S., Feng, P. \& Shi, P. [Study on the epidemic development of COVID-19 in Hubei province by a modified 674 SEIR model]. Zhejiang Da Xие Xие Bao Yi Xue Ban 49, 178-184 (2020).

675 22. Huang, R., Liu, M. \& Ding, Y. Spatial-temporal distribution of COVID-19 in China and its prediction: A data676 driven modeling analysis. J Infect Dev Ctries 14, 246-253 (2020).

677 23. Godio, A., Pace, F. \& Vergnano, A. SEIR Modeling of the Italian Epidemic of SARS-CoV-2 Using 678 Computational Swarm Intelligence. Int J Environ Res Public Health 17, (2020).

679 24. Gatto, M. et al. Spread and dynamics of the COVID-19 epidemic in Italy: Effects of emergency containment 680 measures. Proc. Natl. Acad. Sci. U.S.A. 117, 10484-10491 (2020).

681 25. Hou, C. et al. The effectiveness of quarantine of Wuhan city against the Corona Virus Disease 2019 (COVID19): A well-mixed SEIR model analysis. J. Med. Virol. 92, 841-848 (2020).

683 26. Zhou, T. et al. Preliminary prediction of the basic reproduction number of the Wuhan novel coronavirus 2019nCoV. J Evid Based Med 13, 3-7 (2020).

27. Reno, C. et al. Forecasting COVID-19-Associated Hospitalizations under Different Levels of Social Distancing 686 in Lombardy and Emilia-Romagna, Northern Italy: Results from an Extended SEIR Compartmental Model. $J$ Clin Med 9, (2020).

28. Yu, F. et al. Quantitative Detection and Viral Load Analysis of SARS-CoV-2 in Infected Patients. Clin. Infect. Dis. 71, 793-798 (2020).

29. Rao, S. N., Manissero, D., Steele, V. R. \& Pareja, J. A Narrative Systematic Review of the Clinical Utility of Cycle Threshold Values in the Context of COVID-19. Infect Dis Ther 1-14 (2020) doi:10.1007/s40121-02000324-3. 
30. Alizargar, J. Risk of reactivation or reinfection of novel coronavirus (COVID-19). J. Formos. Med. Assoc. 119, 1123 (2020).

695 31. Batisse, D. et al. Clinical recurrences of COVID-19 symptoms after recovery: viral relapse, reinfection or 696 inflammatory rebound? J. Infect. (2020) doi:10.1016/j.jinf.2020.06.073.

697 32. Deng, W. et al. Primary exposure to SARS-CoV-2 protects against reinfection in rhesus macaques. Science $698 \quad$ (2020) doi:10.1126/science.abc5343.

699 33. Ota, M. Will we see protection or reinfection in COVID-19? Nat. Rev. Immunol. 20, 351 (2020).

700 34. Victor Okhuese, A. Estimation of the Probability of Reinfection With COVID-19 by the Susceptible-Exposed701 Infectious-Removed-Undetectable-Susceptible Model. JMIR Public Health Surveill 6, e19097 (2020).

702 35. Gu, Y. COVID-19 Projections Using Machine Learning. COVID-19 Projections Using Machine Learning 703 https://covid19-projections.com/.

704 36. Stadlbauer, D. et al. Seroconversion of a city: Longitudinal monitoring of SARS-CoV-2 seroprevalence in New 705 York City. medRxiv 2020.06.28.20142190 (2020) doi:10.1101/2020.06.28.20142190.

37. Goodnough, A. \& Shear, M. D. The U.S.'s Slow Start to Coronavirus Testing: A Timeline. The New York Times (2020).

38. Shear, M. D. et al. The Lost Month: How a Failure to Test Blinded the U.S. to Covid-19. The New York Times (2020).

39. Kaplan, S. \& Thomas, K. Despite Promises, Testing Delays Leave Americans 'Flying Blind'. The New York Times (2020).

712 40. de Souza, W. M. et al. Epidemiological and clinical characteristics of the COVID-19 epidemic in Brazil.

$713 \quad$ Nature Human Behaviour 4, 856-865 (2020).

714 41. Mervosh, S. \& Fernandez, M. 'It's Like Having No Testing': Coronavirus Test Results Are Still Delayed. The $715 \quad$ New York Times (2020).

716 42. Gostic, K., Gomez, A. C., Mummah, R. O., Kucharski, A. J. \& Lloyd-Smith, J. O. Estimated effectiveness of 717 symptom and risk screening to prevent the spread of COVID-19. eLife 9, e55570 (2020).

718 43. Zitek, T. The Appropriate Use of Testing for COVID-19. West J Emerg Med 21, 470-472 (2020).

719 44. Bosch, I. et al. Rapid antigen tests for dengue virus serotypes and Zika virus in patient serum. Sci Transl Med $\mathbf{9}$, $720 \quad$ (2017) 
721 45. Massachusetts Department of Public Health. COVID-19 Response Reporting. Mass.gov

722 https://www.mass.gov/info-details/covid-19-response-reporting.

723 46. California Department of Public Health. COVID-19 Cases - California Open Data.

$724 \quad$ https://data.ca.gov/dataset/covid-19-cases.

725 47. California Department of Public Health. COVID-19 Hospital Data - California Open Data.

726 https://data.ca.gov/dataset/covid-19-hospital-data.

727 48. Department of Health and Human Hygiene. COVID-19 Daily Counts of Cases, Hospitalizations, and Deaths |

728 NYC Open Data. https://data.cityofnewyork.us/Health/COVID-19-Daily-Counts-of-Cases-Hospitalizations-

$729 \quad$ an/rc75-m7u3.

730 49. Sao Jose do Rio Preto Public Health Office. COVID-19 Surveillance Data, Sao Jose do Rio Preto.

731 50. New York State Government. Daily Hospitalization Summary by Region. New York Forward

732 https://forward.ny.gov/daily-hospitalization-summary-region.

733 51. Massachusetts General Hospital Institute for Technology Assessment. COVID-19 Simulator - Methodology.

734 https://www.covid19sim.org/images/docs/COVID-19_simulator_methodology_download_20200507.pdf.

735 52. CDC. Coronavirus Disease 2019 (COVID-19). Centers for Disease Control and Prevention

736 https://www.cdc.gov/coronavirus/2019-ncov/cases-updates/commercial-lab-surveys.html (2020).

737 53. SciPy.org — SciPy.org. https://www.scipy.org/.

738 54. NumPy. https://numpy.org/.

739 55. Non-Linear Least-Squares Minimization and Curve-Fitting for Python — Non-Linear Least-Squares

740 Minimization and Curve-Fitting for Python. https://lmfit.github.io/lmfit-py/.

741 56. Matplotlib: Python plotting — Matplotlib 3.3.1 documentation. https://matplotlib.org/.

742 57. Plotly: The front-end for ML and data science models. https://plotly.com/.

745 Acknowledgments

746 General: We thank Professor Lee Gehrke for critical reading of the manuscript. 
Funding: EN is funded by Tufts University DISC Seed Grant. MLN is supported by a FAPESP grant (\#2020/04836-0) and is a CNPq Research Fellow. AFV is supported by a FAPESP Fellow grant (\#18/17647-0). GRFC is supported by a FAPESP Fellow grant (\#20/07419-0). BHGAM is supported by a FAPESP Scholarship (\#19/06572-2). The funders had no role in the design of the study; in the collection, analyses, or interpretation of data; in the writing of the manuscript, or in the decision to publish the results. NS, ARG, AV, GCDS, TMILDS, BHGAM, MMM, GRFC, FQ, AFNR, MLG, ENN, IB, BBH. Funding acquisition: IB, BBH. Investigation: BN, AB, AR, MB, NS, ARG, AV, GCDS, TMILDS, BHGAM, MMM, GRFC, FQ, AFNR, MLG, ENN, IB, BBH. Methodology: BN, AB, AR, MB, NS, ARG, AV, GCDS, TMILDS, BHGAM, MMM, GRFC, FQ, AFNR, MLG, ENN, IB, BBH. Project administration: MLN, IB, BBH. Resources: MLN, IB, BBH. Supervision: MB, MLN, ENN, IB, BBH. Validation: BN, $\mathrm{AB}, \mathrm{AR}, \mathrm{MB}, \mathrm{ENN}, \mathrm{BBH}$. Visualization: BN, AB, AR, MB, AV, ENN, BBH. Writingoriginal draft: AR, $\mathrm{BBH}$. Writing — review and editing: $\mathrm{BN}, \mathrm{AB}, \mathrm{AR}, \mathrm{MB}, \mathrm{NS}, \mathrm{ARG}$, AV, GCDS, TMILDS, BHGAM, MMM, GRFC, FQ, AFNR, MLG, ENN, IB, BBH. Competing interests: $\mathrm{BN}, \mathrm{AB}, \mathrm{AR}, \mathrm{MB}, \mathrm{NS}, \mathrm{AG}, \mathrm{IB}$, and $\mathrm{BBH}$ are employed by or affiliated with E25Bio Inc. (www.e25bio.com), a company that develops diagnostics for epidemic viruses. 
776 Figures and Tables

778 TABLES

779 Table 1. Data summary of direct antigen rapid test (DART) for detection of SARS-CoV-2

780 nucleocapsid protein and DART for detection of SARS-CoV-2 spike glycoprotein

781 performance in comparison to qRT-PCR results. Sensitivity, specificity, Positive predicative

782 value, (PPV) negative predictive value (NPV), prevalence, and overall agreement are calculated 783 for increasing PCR cycle threshold $(\mathrm{Ct})$ values.

784 


\begin{tabular}{|c|c|c|}
\hline \\
\hline 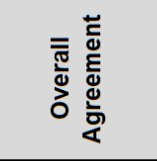 & 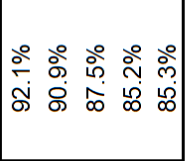 & 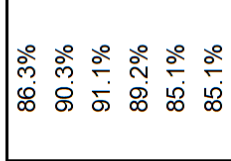 \\
\hline 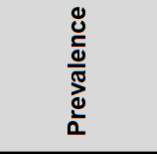 & 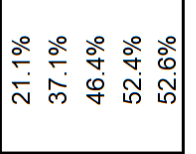 & 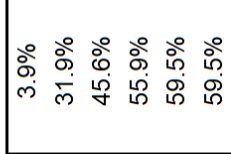 \\
\hline 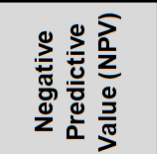 & 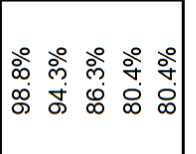 & 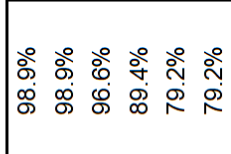 \\
\hline 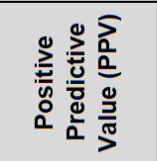 & 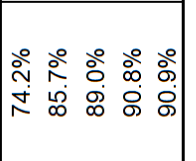 & 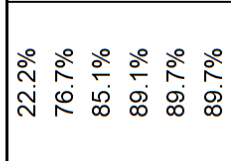 \\
\hline 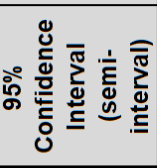 & 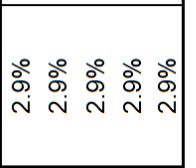 & 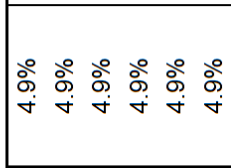 \\
\hline 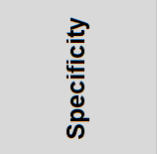 & 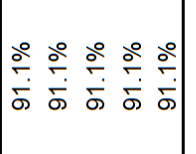 & 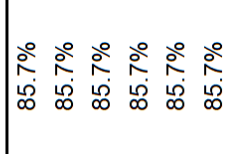 \\
\hline 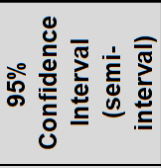 & 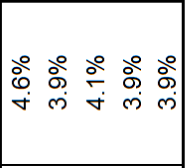 & 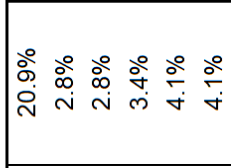 \\
\hline 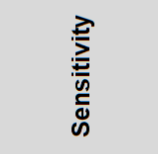 & 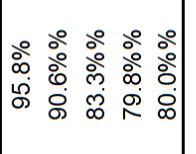 & 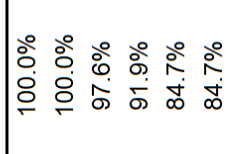 \\
\hline 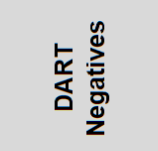 & ৪ ৪ ৪ ৪ ৪ & 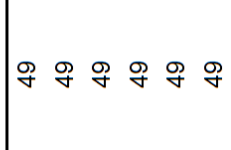 \\
\hline 紫竞 & 공 & 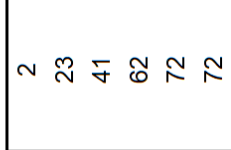 \\
\hline 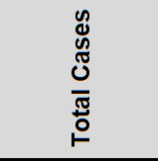 & 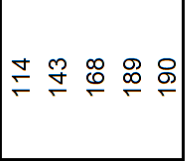 & 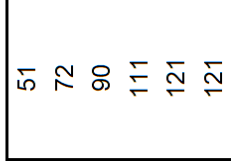 \\
\hline 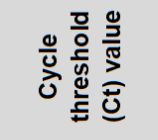 & 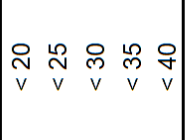 & 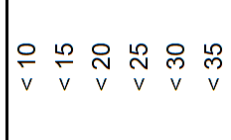 \\
\hline & 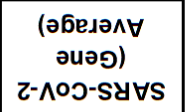 & 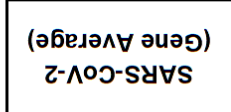 \\
\hline & \begin{tabular}{|c|} 
(u!ə̧одd \\
p!sdeכoә|эnu) \\
$14 \forall \square$ \\
\end{tabular} & 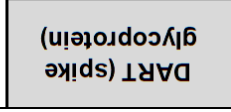 \\
\hline
\end{tabular}


790 Table 2. Clinical validation summary for the direct antigen rapid test (DART) for SARS-

791 CoV-2 nucleocapsid protein evaluated using 190 retrospectively collected patient nasal

792 swab specimens.

793

\begin{tabular}{|c|c|c|c|c|c|c|c|c|}
\hline \multicolumn{9}{|c|}{ All Data Summary } \\
\hline & & \multicolumn{2}{|c|}{$\begin{array}{c}\text { qRT-PCR } \\
\text { (gene average) }\end{array}$} & \multirow[b]{2}{*}{ Total } & \multirow[b]{2}{*}{ Sensitivity } & \multirow[b]{2}{*}{$80.0 \%$} & \multicolumn{2}{|c|}{$\begin{array}{l}\text { 95\% Confidence } \\
\text { Interval }\end{array}$} \\
\hline & & + & $=$ & & & & $76.1 \%$ & $83.9 \%$ \\
\hline \multirow{2}{*}{ 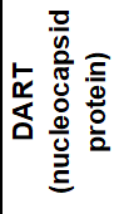 } & + & 80 & 8 & 88 & Specificity & $91.1 \%$ & $88.2 \%$ & $94.0 \%$ \\
\hline & - & 20 & 82 & 102 & $\begin{array}{l}\text { Positive } \\
\text { Predictive } \\
\text { Value }\end{array}$ & $90.9 \%$ & $87.9 \%$ & $93.9 \%$ \\
\hline \multicolumn{2}{|r|}{ Total } & 100 & 90 & 190 & $\begin{array}{c}\text { Negative } \\
\text { Predictive } \\
\text { Value }\end{array}$ & $80.4 \%$ & $76.6 \%$ & $84.2 \%$ \\
\hline & & & & & Prevalence & $52.6 \%$ & $47.8 \%$ & $57.5 \%$ \\
\hline & & & & & $\begin{array}{c}\text { Overall } \\
\text { Agreement }\end{array}$ & $85.3 \%$ & $82.8 \%$ & $87.8 \%$ \\
\hline
\end{tabular}


802 Table 3. Clinical validation summary for the SARS-CoV-2 direct antigen rapid test

803 (DART) for SARS-SoC-2 spike glycoprotein evaluated using 121 retrospectively collected

804 patient nasopharyngeal swab specimens.

805

806

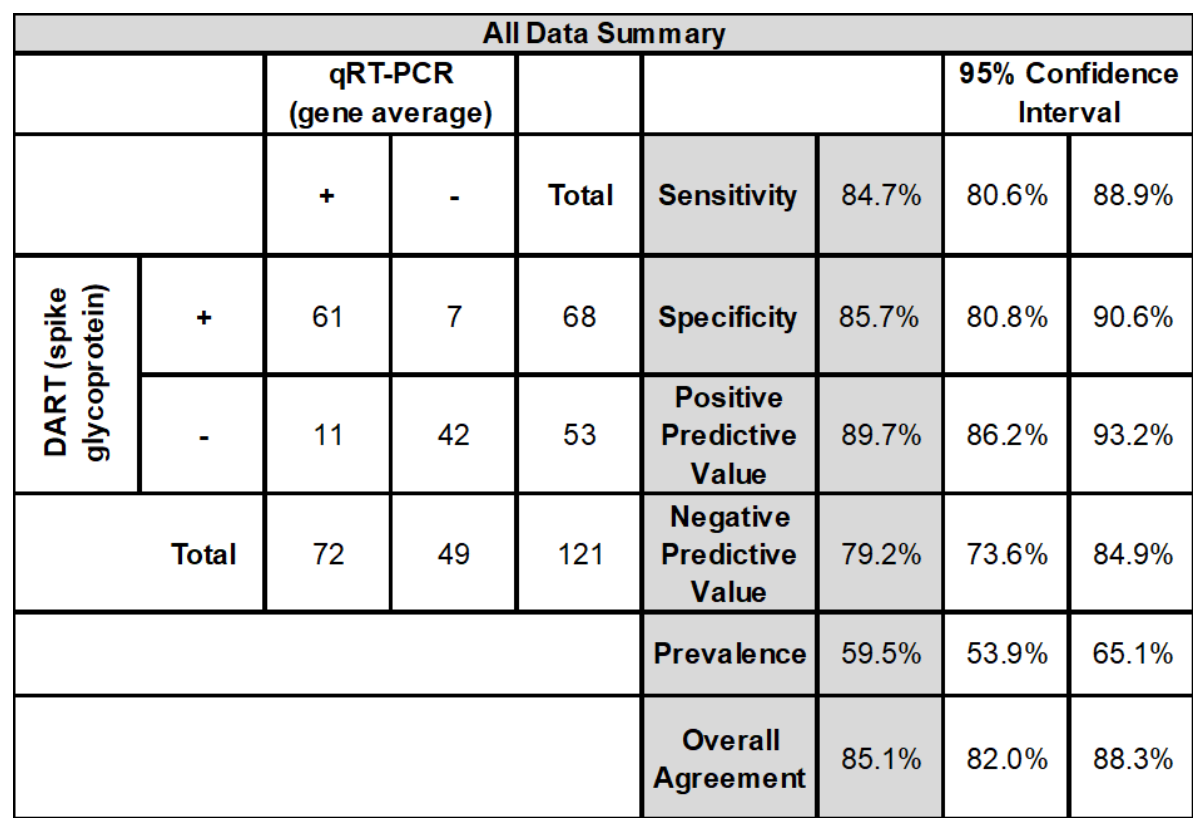

808

809

810

811

812

813

814

815

816

817 


\begin{tabular}{|c|c|c|c|c|}
\hline Parameter & \multicolumn{4}{|l|}{ Details \& Statistics } \\
\hline \multirow{5}{*}{$\alpha$} & \multirow{5}{*}{$\begin{array}{l}\alpha \text { is the probability that an interaction between an undetected infected person and } \\
\text { an uninfected person results in a new infection, divided by the average number of } \\
\text { uninfected people an undetected infected person comes into contact with on a } \\
\text { given day. } \alpha \text { is estimated from the data. }\end{array}$} & & Mean & St. Dev. \\
\hline & & MA & 0.088 & 0.051 \\
\hline & & LA & 0.090 & 0.034 \\
\hline & & NYC & 0.067 & 0.042 \\
\hline & & SJRP & 0.121 & 0.042 \\
\hline$\eta$ & \multicolumn{4}{|c|}{$\begin{array}{l}\eta \text { is the probability that an interaction between an infected person and an uninfected person results in a new } \\
\text { infection, divided by the average number of uninfected people a detected infected person comes into contact } \\
\text { with on a given day. } \eta=0.01 \cdot \alpha \\
\text { The constant relating } \eta, \alpha \text { accounts for a small but nonzero transmission due to the quarantined (detected) } \\
\text { infected population. This value was chosen to be small, assuming a quarantined individual will only infect } \\
\text { others with low probability. }\end{array}$} \\
\hline \multirow[t]{5}{*}{$v$} & \multirow{5}{*}{$\begin{array}{l}\nu \text { is the probability that a symptomatic undetected individual is diagnosed on a } \\
\text { given day. } v \text { is estimated from the data. } v \text { is multiplied by sensitivity (assume } \\
\text { benchmark sensitivity } 100 \% \text { for PCR, as used when fitting). }\end{array}$} & & Mean & St. Dev. \\
\hline & & MA & 0.006 & 0.005 \\
\hline & & LA & 0.011 & 0.006 \\
\hline & & NYC & 0.0056 & 0.002 \\
\hline & & SJRP & 0.015 & 0.007 \\
\hline$\epsilon$ & \multicolumn{4}{|c|}{$\begin{array}{l}\epsilon \text { is the probability that an asymptomatic undetected infected individual is diagnosed on a given day. } \epsilon=0 \\
\text { while fitting (during PCR symptomatic testing). } \epsilon=\text { (sensitivity/days between tests) when the rapid testing } \\
\text { strategy is activated. }\end{array}$} \\
\hline$\lambda$ & \multicolumn{4}{|c|}{$\begin{array}{l}\lambda \text { is the probability that an undetected infected individual transitions to the recovered state on a given day. } \lambda= \\
1 / 14 \text {, or the inverse of average recovery time }{ }^{51} \text {. }\end{array}$} \\
\hline \multirow[t]{5}{*}{$\mu$} & \multirow{5}{*}{$\begin{array}{l}\mu \text { is the probability that an infected individual develops severe symptoms on a } \\
\text { given day and transitions into the hospitalized state. The flow from } D \text { to } H \text { is } \\
\text { assumed to be independent of the ratio } I / D \text {, but comes only from the detected } \\
\text { infected population, hence why it is multiplied by }(I+D) / D . \mu \text { is estimated from } \\
\text { the data. }\end{array}$} & & Mean & St. Dev. \\
\hline & & MA & 0.0013 & $9.5 \mathrm{e}-4$ \\
\hline & & LA & 0.0016 & $2.4 \mathrm{e}-4$ \\
\hline & & NYC & 0.0011 & 6.6e-4 \\
\hline & & SJRP & 0.0018 & $8.0 \mathrm{e}-4$ \\
\hline
\end{tabular}




\begin{tabular}{|c|c|c|c|c|}
\hline$\rho$ & \multicolumn{4}{|c|}{$\begin{array}{l}\rho \text { is the probability that a detected infected individual transitions to the recovered state on a given day. } \\
\rho=1 / 14 \text {, or the inverse of the average recovery time }{ }^{51} \text {. }\end{array}$} \\
\hline$\sigma$ & \multicolumn{4}{|c|}{$\begin{array}{l}\sigma \text { is the probability that a hospitalized individual transitions to the recovered state on a given day. } \sigma=1 / 11 \text {, } \\
\text { or the inverse of the average recovery time for a hospitalized individual }{ }^{51} \text {. }\end{array}$} \\
\hline \multirow[t]{5}{*}{$\tau$} & \multirow{5}{*}{$\begin{array}{l}\tau \text { is the probability that a hospitalized individual expires on a given day. } \tau \text { is } \\
\text { estimated from the data. }\end{array}$} & & Mean & St. Dev. \\
\hline & & MA & 0.034 & 0.012 \\
\hline & & LA & 0.016 & 0.004 \\
\hline & & NYC & 0.036 & 0.034 \\
\hline & & SJRP & 0.032 & 0.045 \\
\hline$\gamma$ & \multicolumn{4}{|c|}{$\begin{array}{l}\gamma \text { is the probability of entering either of the quarantine states on a given day from either the Susceptible or } \\
\text { Recovered populations. } \gamma=0 \text { while fitting (during PCR symptomatic testing). } \gamma=(1-\text { specificity) } \times \\
\text { (1/days between tests) when the rapid testing strategy is activated. }\end{array}$} \\
\hline$\psi$ & \multicolumn{4}{|c|}{$\begin{array}{l}\psi \text { is the probability that an individual exits quarantine on a given day. } \psi=1 / 14 \text {, or the inverse of the } \\
\text { quarantine period for fixed length quarantine. }\end{array}$} \\
\hline Parameter & \multicolumn{4}{|l|}{ Details \& Statistics } \\
\hline \multirow{5}{*}{$\mid \alpha$} & \multirow{5}{*}{$\begin{array}{l}\mid \alpha \text { is the probability that an interaction between an undetected infected person } \\
\text { and an uninfected person results in a new infection, divided by the average } \\
\text { number of uninfected people an undetected infected person comes into contact } \\
\text { with on a given day. } \mid \alpha \text { is estimated from the data. }\end{array}$} & & Mean & St. Dev. \\
\hline & & MA & 0.088 & 0.051 \\
\hline & & LA & 0.090 & 0.034 \\
\hline & & NYC & 0.067 & 0.042 \\
\hline & & SJRP & 0.121 & 0.042 \\
\hline $\bar{\eta}$ & \multicolumn{4}{|c|}{$\begin{array}{l}\eta \text { is the probability that an interaction between an infected person and an uninfected person results in a new } \\
\text { infection, divided by the average number of uninfected people a detected infected person comes into contact } \\
\text { with on a given day. } \eta=0.01 \cdot a \\
\text { The constant relating } \eta, a \text { accounts for a small but nonzero transmission due to the quarantined (detected) } \\
\text { infected population. This value was chosen to be small, assuming a quarantined individual will only infect } \\
\text { others with low probability. }\end{array}$} \\
\hline \multirow[t]{4}{*}{$h$} & \multirow{4}{*}{$\begin{array}{l}W \text { is the probability that a symptomatic undetected individual is diagnosed on a } \\
\text { given day. } \mid v \text { is estimated from the data. } \mid v \text { is multiplied by sensitivity (assume } \\
\text { benchmark sensitivity } 100 \% \text { for PCR, as used when fitting). }\end{array}$} & & Mean & St. Dev. \\
\hline & & MA & 0.006 & 0.005 \\
\hline & & LA & 0.011 & 0.006 \\
\hline & & NYC & 0.0056 & 0.002 \\
\hline
\end{tabular}




\begin{tabular}{|c|c|c|c|c|}
\hline & & SJRP & 0.015 & 0.007 \\
\hline$E$ & \multicolumn{4}{|c|}{$\begin{array}{l}\epsilon \text { is the probability that an asymptomatic undetected infected individual is diagnosed on a given day. } \overline{\epsilon=0} \\
\text { while fitting (during PCR symptomatic testing). } \epsilon=\text { (sensitivity/days between tests) when the rapid testing } \\
\text { strategy is activated. }\end{array}$} \\
\hline$\lambda$ & \multicolumn{4}{|c|}{$\begin{array}{l}\lambda \text { is the probability that an undetected infected individual transitions to the recovered state on a given day. } \\
\lambda=1 / 14 \text {, or the inverse of average recovery time }{ }^{51} \text {. }\end{array}$} \\
\hline \multirow[t]{5}{*}{$\mu$} & \multirow{5}{*}{ 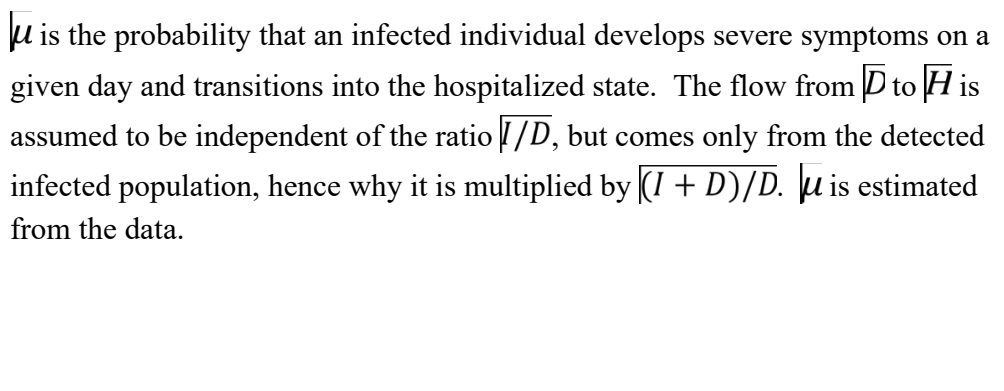 } & & Mean & St. Dev. \\
\hline & & MA & 0.0013 & $9.5 \mathrm{e}-4$ \\
\hline & & LA & 0.0016 & $2.4 \mathrm{e}-4$ \\
\hline & & NYC & 0.0011 & $6.6 \mathrm{e}-4$ \\
\hline & & SJRP & 0.0018 & $8.0 \mathrm{e}-4$ \\
\hline$\rho$ & \multicolumn{4}{|c|}{$\begin{array}{l}\rho \text { is the probability that a detected infected individual transitions to the recovered state on a given day. } \\
\rho=1 / 14 \text {, or the inverse of the average recovery time }{ }^{51} \text {. }\end{array}$} \\
\hline $\bar{\sigma}$ & \multicolumn{4}{|c|}{$\begin{array}{l}\sigma \text { is the probability that a hospitalized individual transitions to the recovered state on a given day. } \overline{\sigma=1 / 11} \\
\text {, or the inverse of the average recovery time for a hospitalized individual }{ }^{51} \text {. }\end{array}$} \\
\hline \multirow[t]{5}{*}{$\bar{\tau}$} & \multirow{5}{*}{$\begin{array}{l}\mp \text { is the probability that a hospitalized individual expires on a given day. }\lceil\boldsymbol{\tau} \text { is } \\
\text { estimated from the data. }\end{array}$} & & Mean & St. Dev. \\
\hline & & MA & 0.034 & 0.012 \\
\hline & & LA & 0.016 & 0.004 \\
\hline & & NYC & 0.036 & 0.034 \\
\hline & & SJRP & 0.032 & 0.045 \\
\hline $\bar{b}$ & \multicolumn{4}{|c|}{$\begin{array}{l}\mid y \text { is the probability of entering either of the quarantine states on a given day from either the Susceptible or } \\
\text { Recovered populations. } y=0 \text { while fitting (during PCR symptomatic testing). } y=(1-\text { specificity }) \times(1 / \\
\text { days between tests } \sqrt{\text { when the rapid testing strategy is activated. }}\end{array}$} \\
\hline $\bar{\psi}$ & \multicolumn{4}{|c|}{$\begin{array}{l}\sqrt{\psi} \text { is the probability that an individual exits quarantine on a given day. } \psi=1 / 14 \text {, or the inverse of the } \\
\text { quarantine period for fixed length quarantine. }\end{array}$} \\
\hline
\end{tabular}


827 infection in SIDHRE-Q model. Q-U, quarantine uninfected; S, susceptible (uninfected); I,

828 infected undetected (pre-testing and infected); D, infected detected (infection diagnosis through

829 testing); $\mathbf{H}$, hospitalized (infected with life threatening symptom progression); $\mathbf{R}$, recovered

830 (healed); E, extinct (dead); and Q-R, quarantine recovered (healed but in quarantine by false

831 positive testing).

832

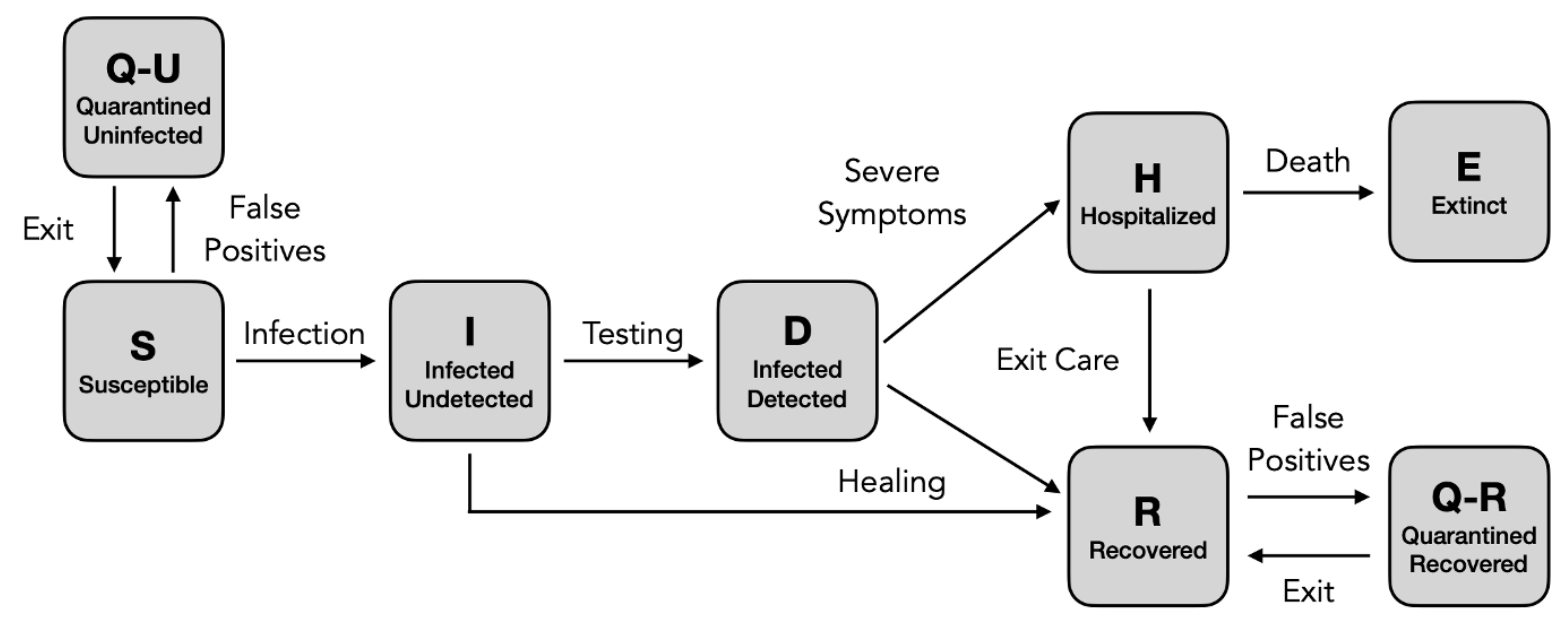


842 Fig. 2. COVID-19 Outcomes in 3 US Regions and Brazil as a result of Frequent Rapid

843 Testing Protocol using the SIDHRE- $Q$ Model. The Cumulative Detected Infected,

844 Hospitalized, Deceased, Active Infections, Recovered, and Quarantined are modeled over 105

845 days (top to bottom) using reported data from 4 global regions: Massachusetts, Los Angeles,

846 New York City, and São José do Rio Preto in Brazil (left to right). The COVID-19 population

847 spread and outcomes are modeled under a Rapid Testing Protocol (sensitivity $80 \%$, specificity

$84890 \%$ ) with variable testing frequencies ranging from 1-21 days between tests. This protocol is

849 compared to a symptom-based Rapid Testing protocol and a symptom-based PCR protocol. 

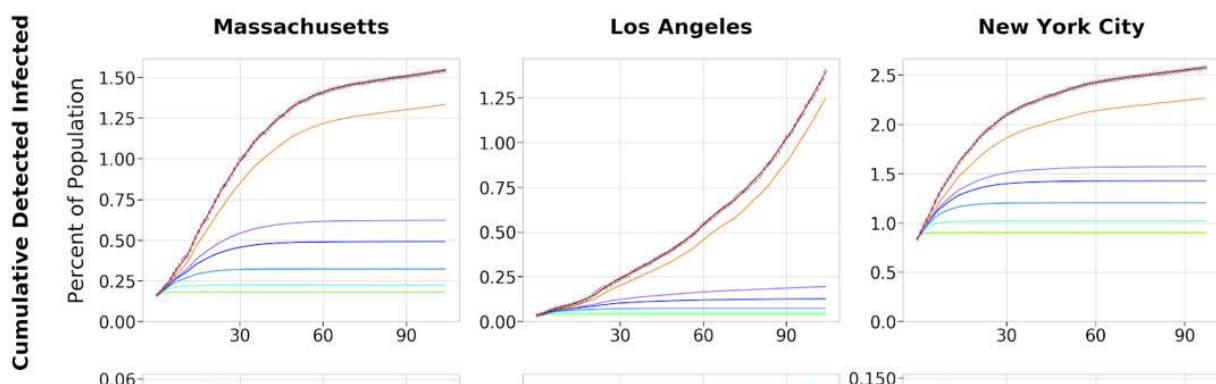

São José do Rio Preto, Brazil
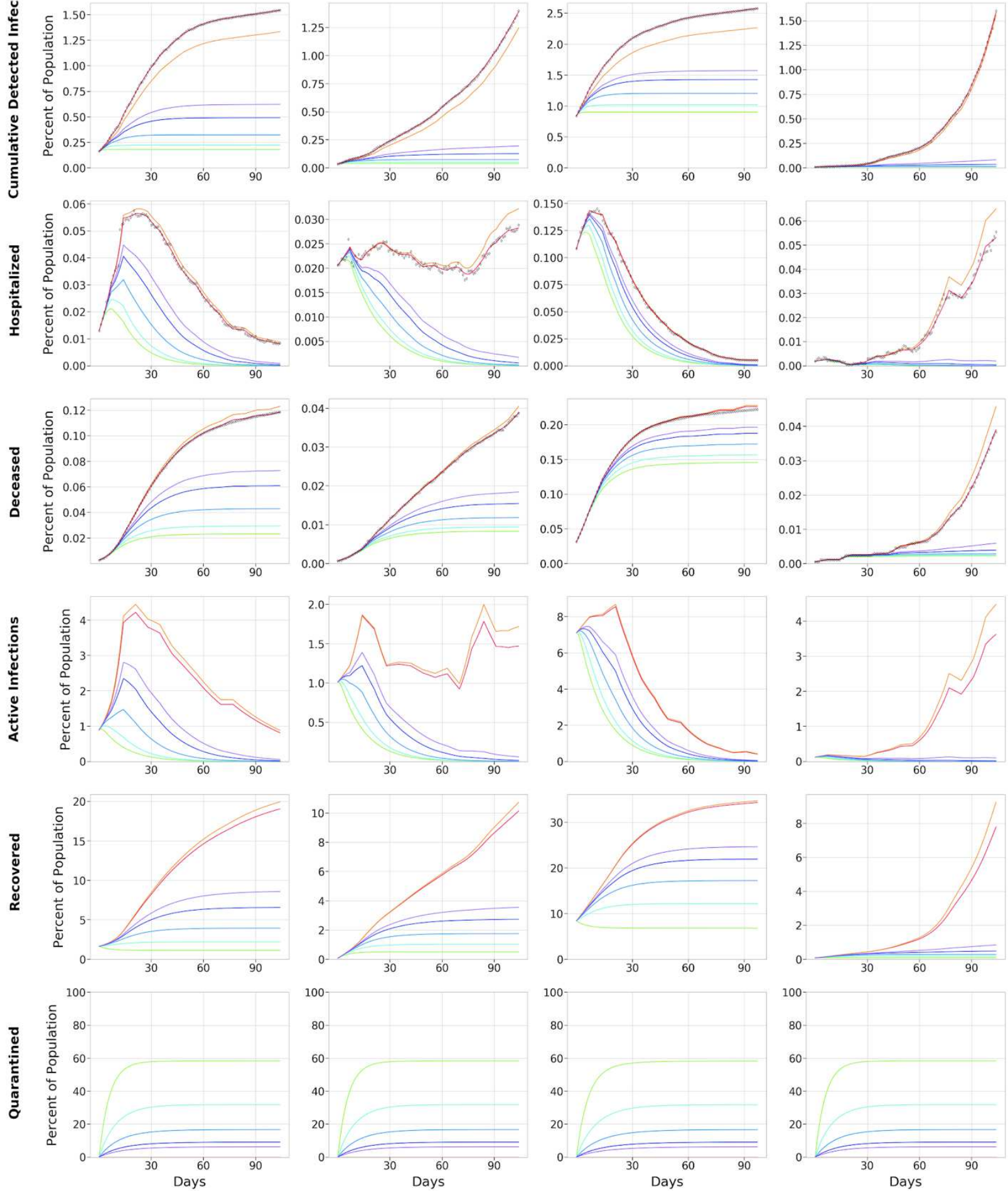

Symptomatic Testing
(PCR) Symptomatic Testing
(Rapid Test)

Sensitivity: $\mathbf{8 0 . 0} \%$ Specificity: $\mathbf{9 0 . 0} \%$

: $80.0 \%$ Specificity: $90.0 \%$ 
854 increasing frequency under the SIDHRE-Q Model. The Cumulative Infections, Maximum

855 Simultaneously Hospitalized, and Deceased populations are modeled for Massachusetts, Los

856 Angeles, New York City, and São José do Rio Preto in Brazil with a 90\% test specificity.

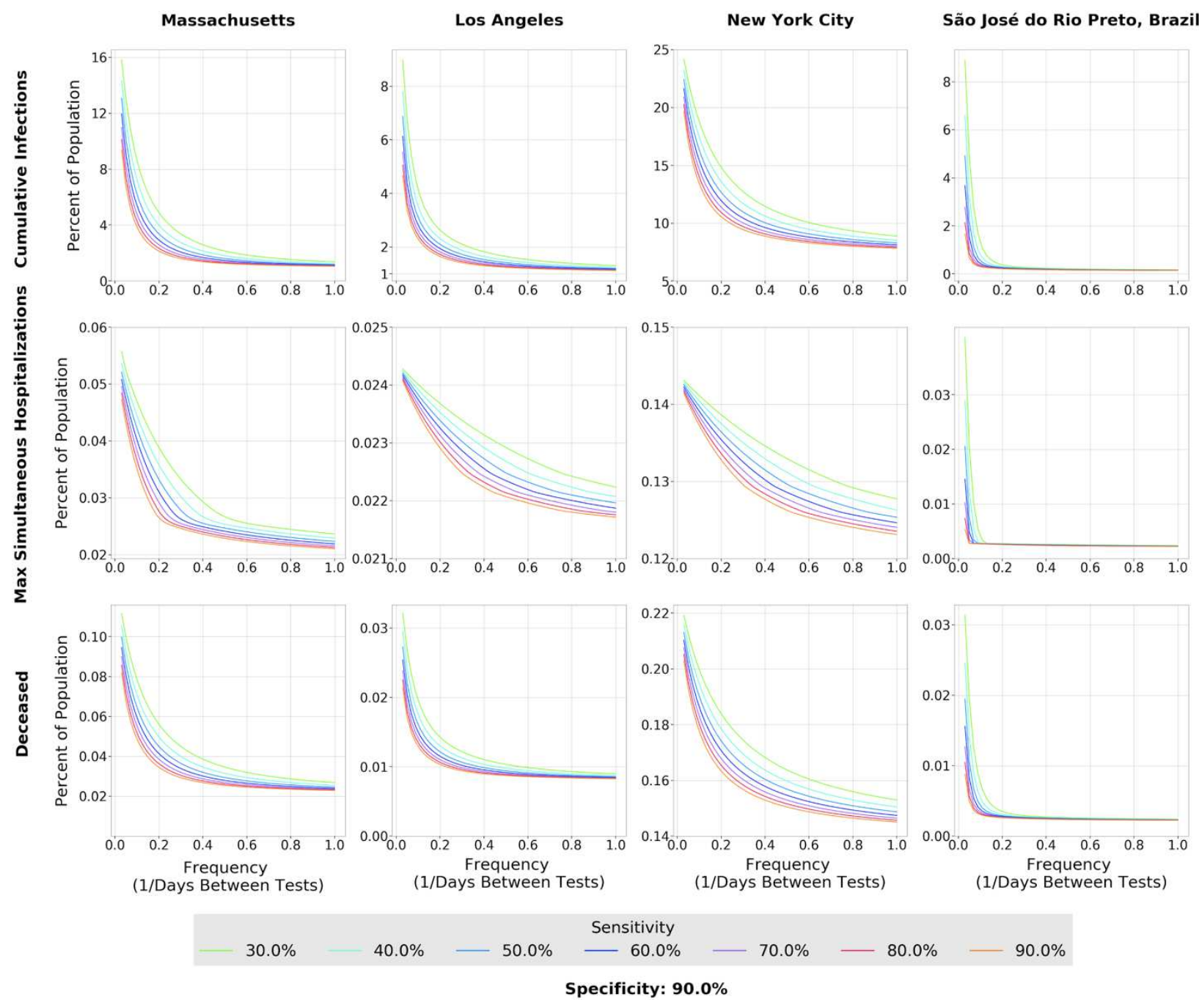


866 Fig. 4. Effect of County Based Rapid Testing strategy on COVID-19 outcomes in

867 California. This protocol varies testing frequency in accordance to the number of recorded 868 cases; the threshold for number of active infections which, if reached, signals to commence 869 everyday testing (the highest frequency considered). A Rapid Test with an $80 \%$ sensitivity and

$87090 \%$ sensitivity versus is used in this deployment strategy. Shown is the total cost per person per 871 day versus the cumulative infections, maximum simultaneously hospitalized, and cumulative 872 deaths with varied thresholds for all of CA is shown. The County Based Rapid Testing strategy

873 is compared to uniform testing, which distributes the same number of total tests used in the 874 county strategy, albeit evenly across each county. The effects of uniform testing are modeled for 875 both a Rapid Testing protocol and a qRT-PCR protocol (A). The effects of County Based Rapid 876 Test Protocol and Uniform PCR Protocol on active infected detected population over time in CA 877 are shown (B). The legend denotes the thresholds at which testing frequency is determined, the 878 testing frequencies, the percent of CA population under the strategy, and the cost per person per 879 day. 
Sensitivity: $80.0 \%$, Specificity: $90.0 \%$
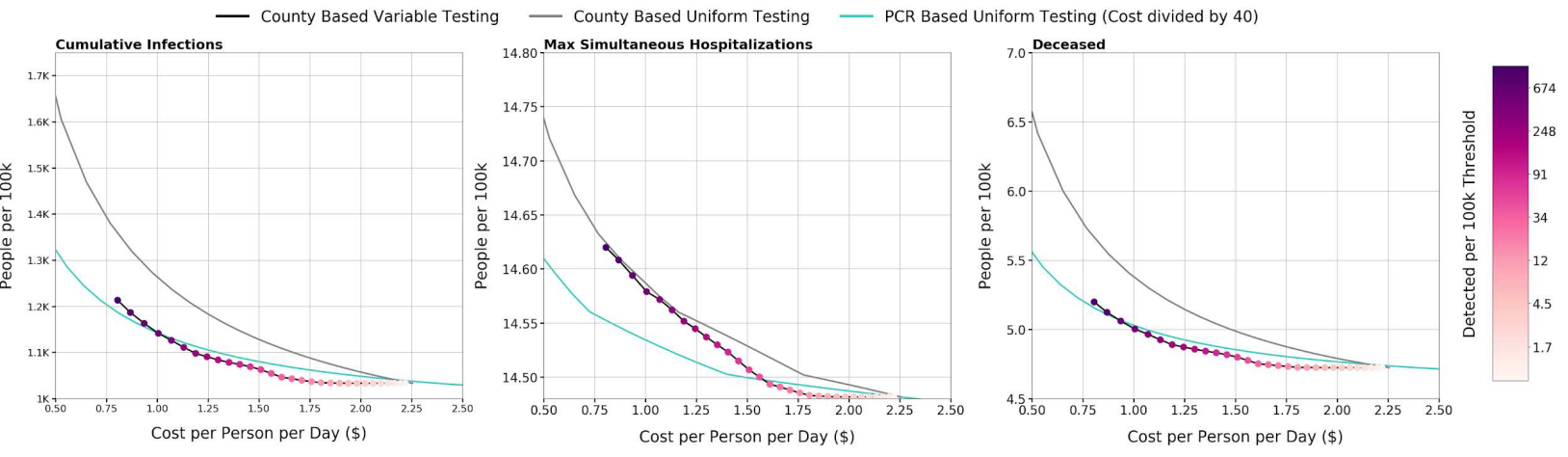

887

888

(B)
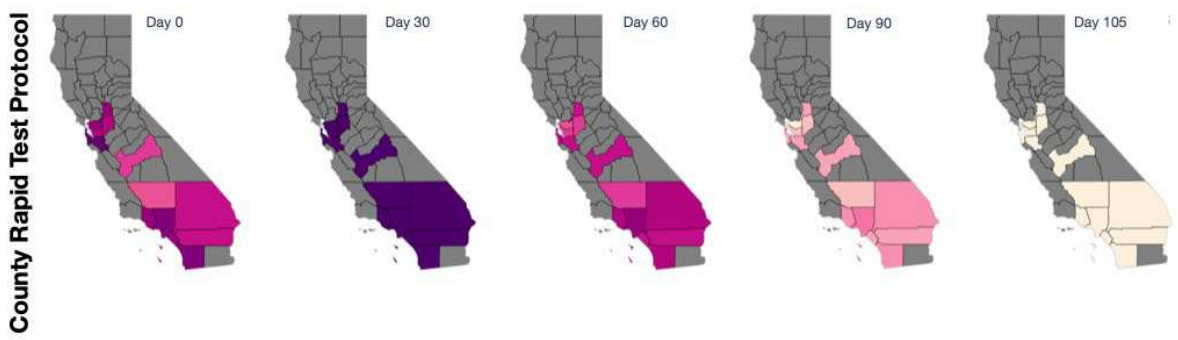

Detected per 100k | Days Between Tests - No Testing Done

$0.0-0.8|14=6.2-8.8| 7$

$=0.8 \cdot 1.1|13=8.8 \cdot 12| 6$

$-1.1-1.6 \mid 12=12-18$ |

$=1.6-2.2|11=18-25| 4$

- $2.2 .31110=18-2514$

$=3.1-4.419$

$=3.1-4.419=-35.5012$

- $4.4-6.218=50-1 \mathrm{~K} \mid 1$

Test Sensitivity: 0.8

Test Specificity: 0.9

Percent of CA Under Protocol: $84.7 \%$

Cost per Person per Day: $\$ 1.53$
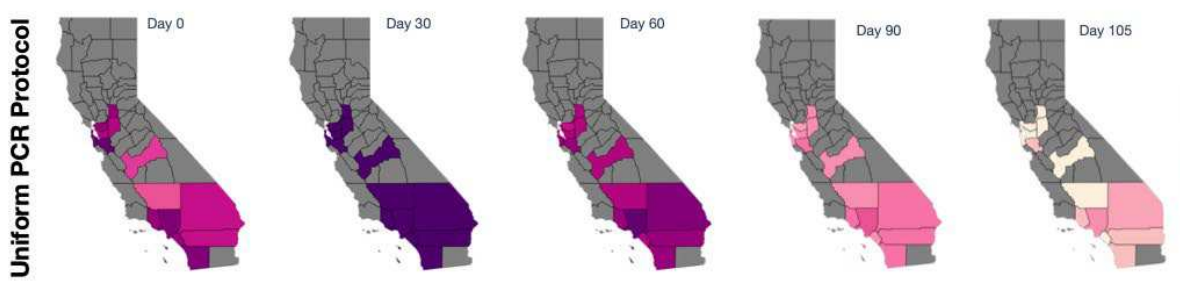

Detected per 100k | Days Between Tests

- No Testing Done

$0.0-0.8|7=6.2-8.8| 7$

$=0.8-1.1|7=8.8-12| 7$

$=1.1-1.6|7 \quad 12-18| 7$

$=1.6-2.217=18-2517$

- 2.2-3.117 $25-3517$

- 3.1-4.417 $35-5017$

$=4.4-6.217=50-1 \mathrm{~K} \mid 7$

PCR Based Uniform Testing

Percent of CA Under Protocol: $84.7 \%$ Cost per Person per Day: $\$ 14.27$ 


\section{Figures}

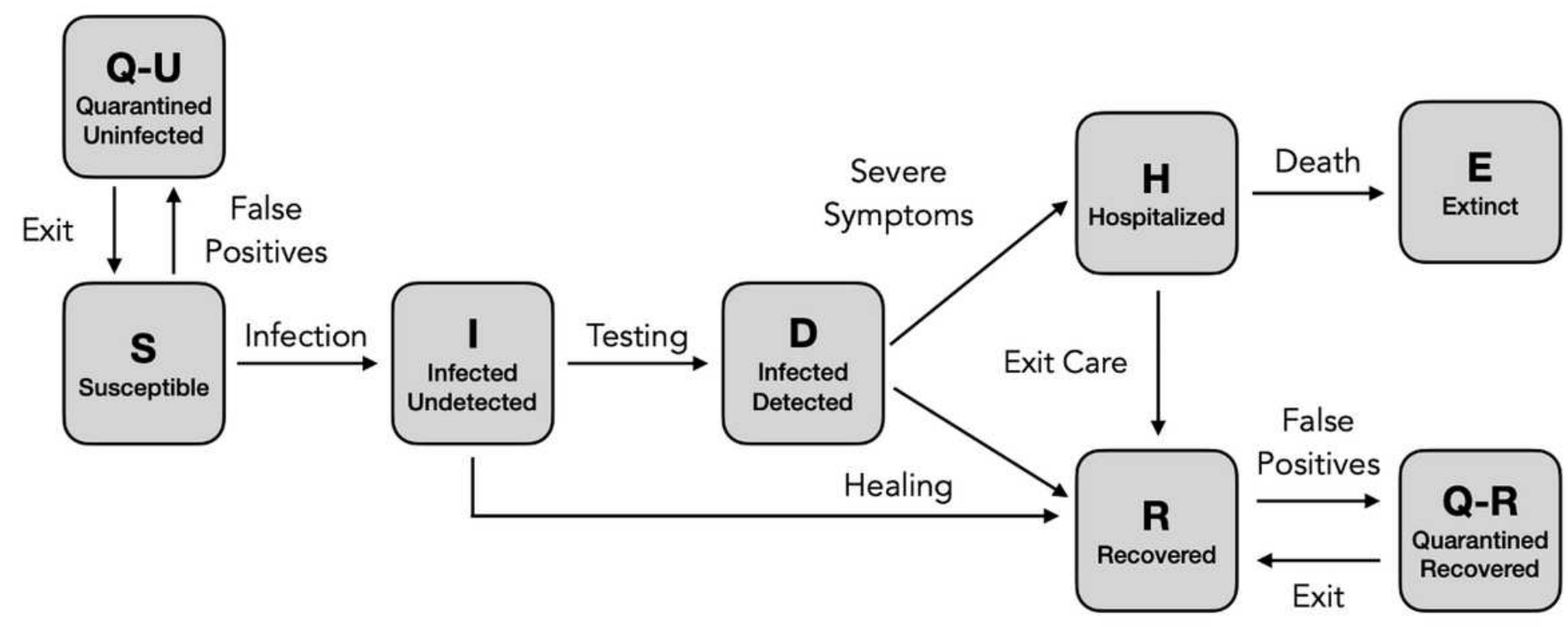

\section{Figure 1}

Graphical scheme displaying the relationships between the stages of quarantine and infection in SIDHREQ model. Q-U, quarantine uninfected; S, susceptible (uninfected); I, infected undetected (pre-testing and infected); $\mathrm{D}$, infected detected (infection diagnosis through testing); $\mathrm{H}$, hospitalized (infected with life threatening symptom progression); $\mathrm{R}$, recovered (healed); $\mathrm{E}$, extinct (dead); and Q-R, quarantine recovered (healed but in quarantine by false positive testing). 

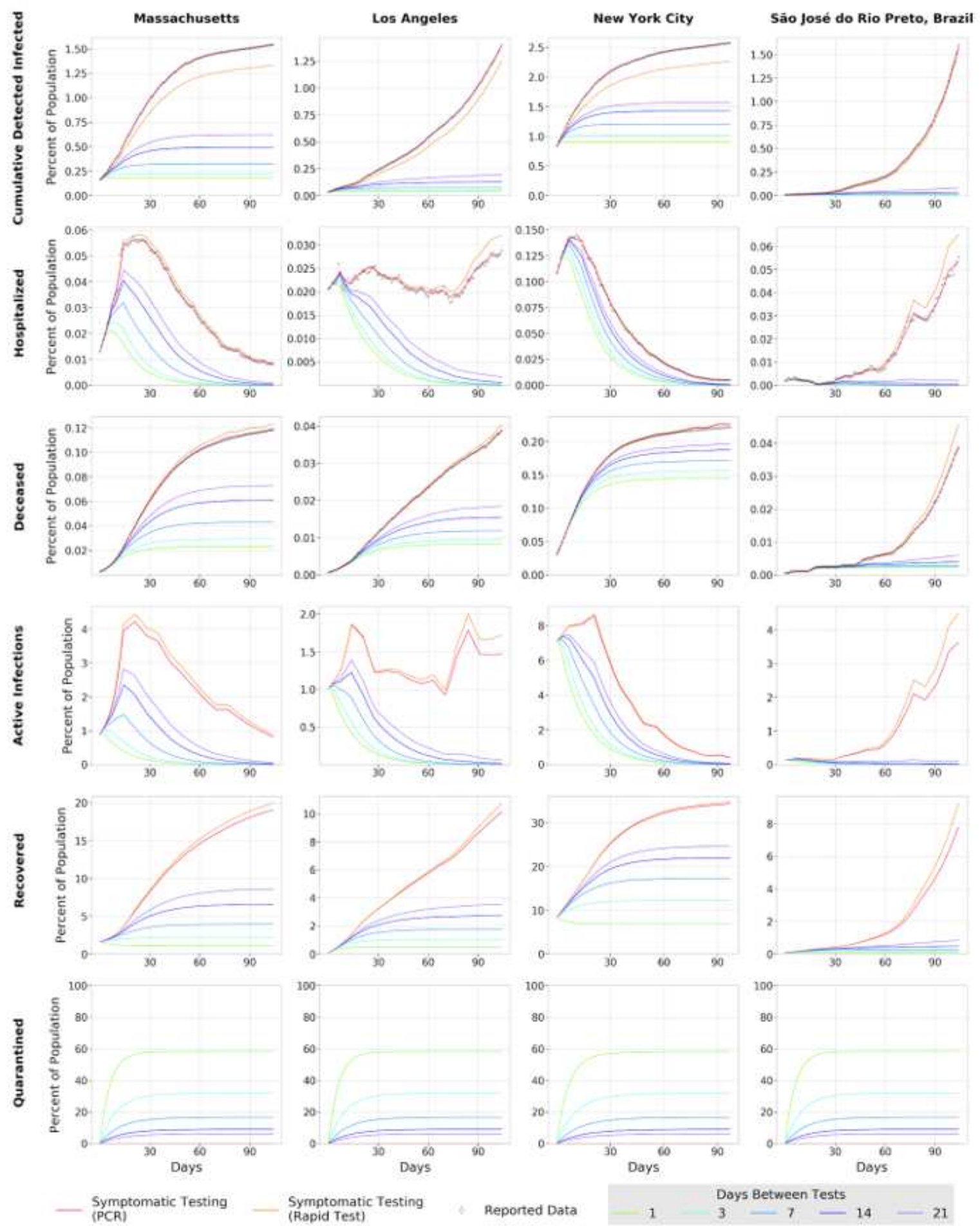

(Rapid Test)
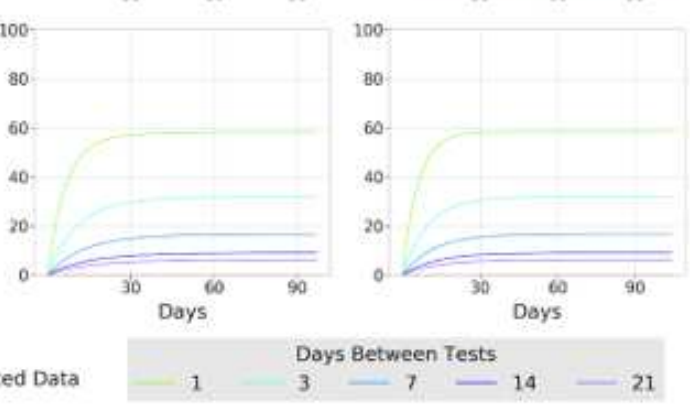

Sensitivity: $80.0 \%$ Specificity: $90.0 \%$

\section{Figure 2}

COVID-19 Outcomes in 3 US Regions and Brazil as a result of Frequent Rapid Testing Protocol using the SIDHRE-Q Model. The Cumulative Detected Infected, Hospitalized, Deceased, Active Infections, Recovered, and Quarantined are modeled over 105 days (top to bottom) using reported data from 4 global regions: Massachusetts, Los Angeles, New York City, and São José do Rio Preto in Brazil (left to right). The COVID19 population spread and outcomes are modeled under a Rapid Testing Protocol (sensitivity 80\%, 
specificity $90 \%$ ) with variable testing frequencies ranging from 1-21 days between tests. This protocol is compared to a symptom-based Rapid Testing protocol and a symptom-based PCR protocol.

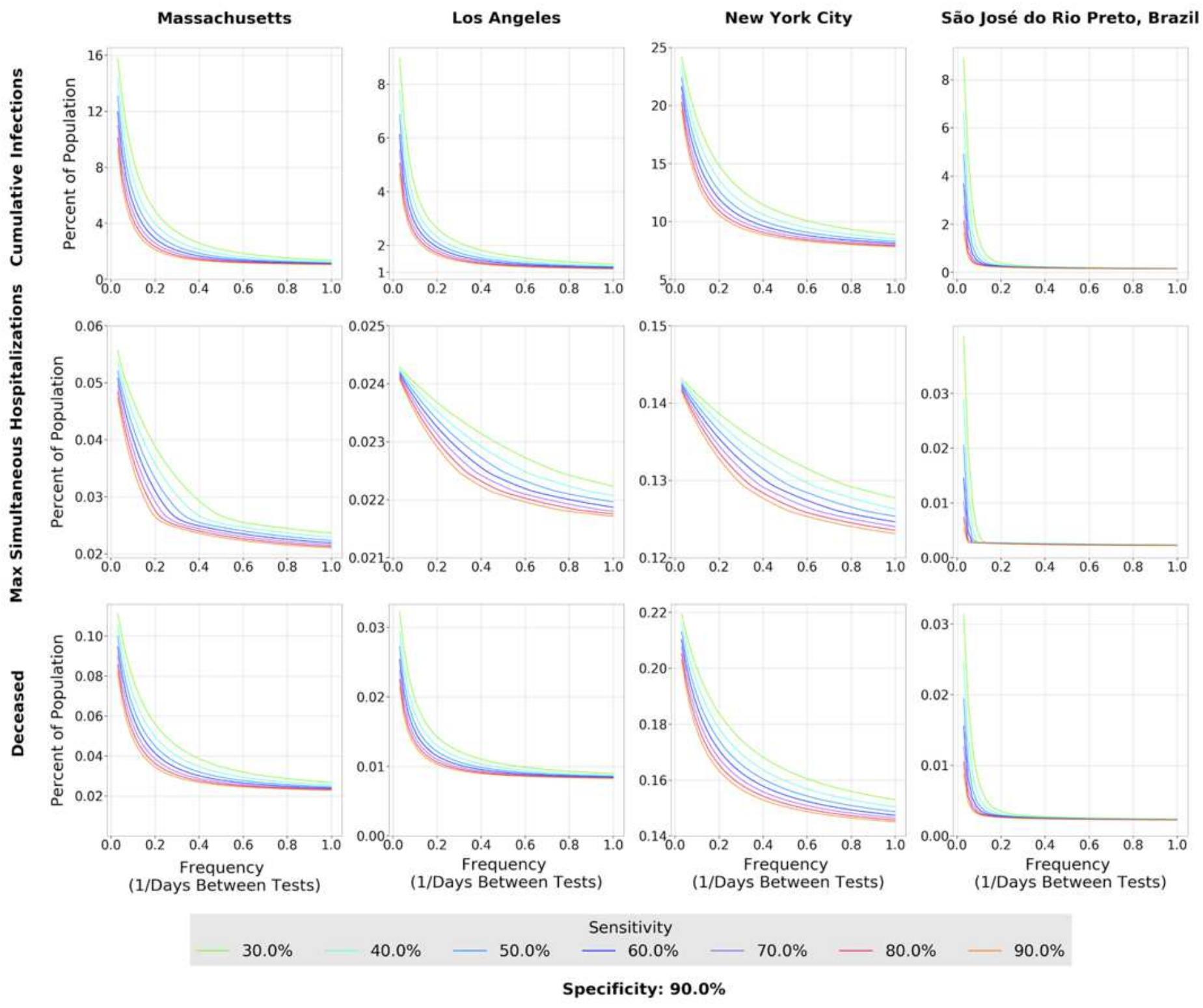

Figure 3

Effect of Rapid Testing Protocol under variable testing sensitivities (30\%-90\%) and increasing frequency under the SIDHRE-Q Model. The Cumulative Infections, Maximum Simultaneously Hospitalized, and Deceased populations are modeled for Massachusetts, Los Angeles, New York City, and São José do Rio Preto in Brazil with a $90 \%$ test specificity. 
(A)

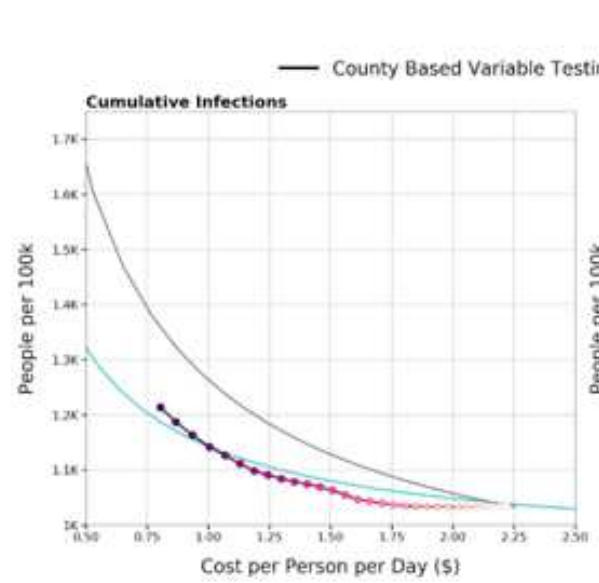

Sensitivity: $80,0 \%, 5$ pecificity: $90.0 \%$
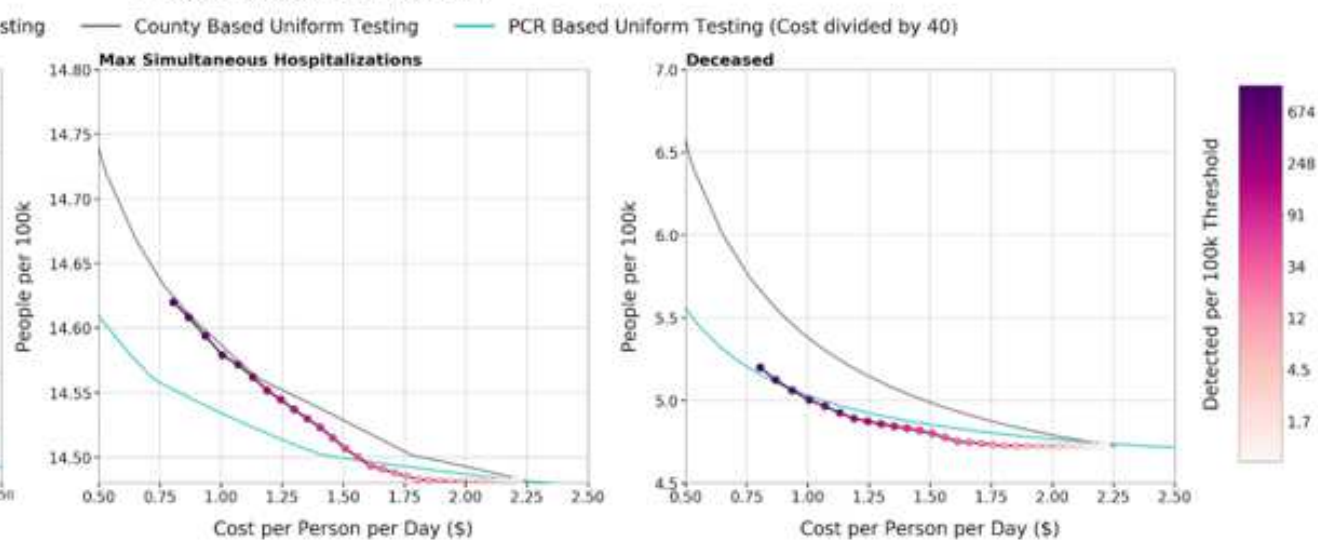

(B)
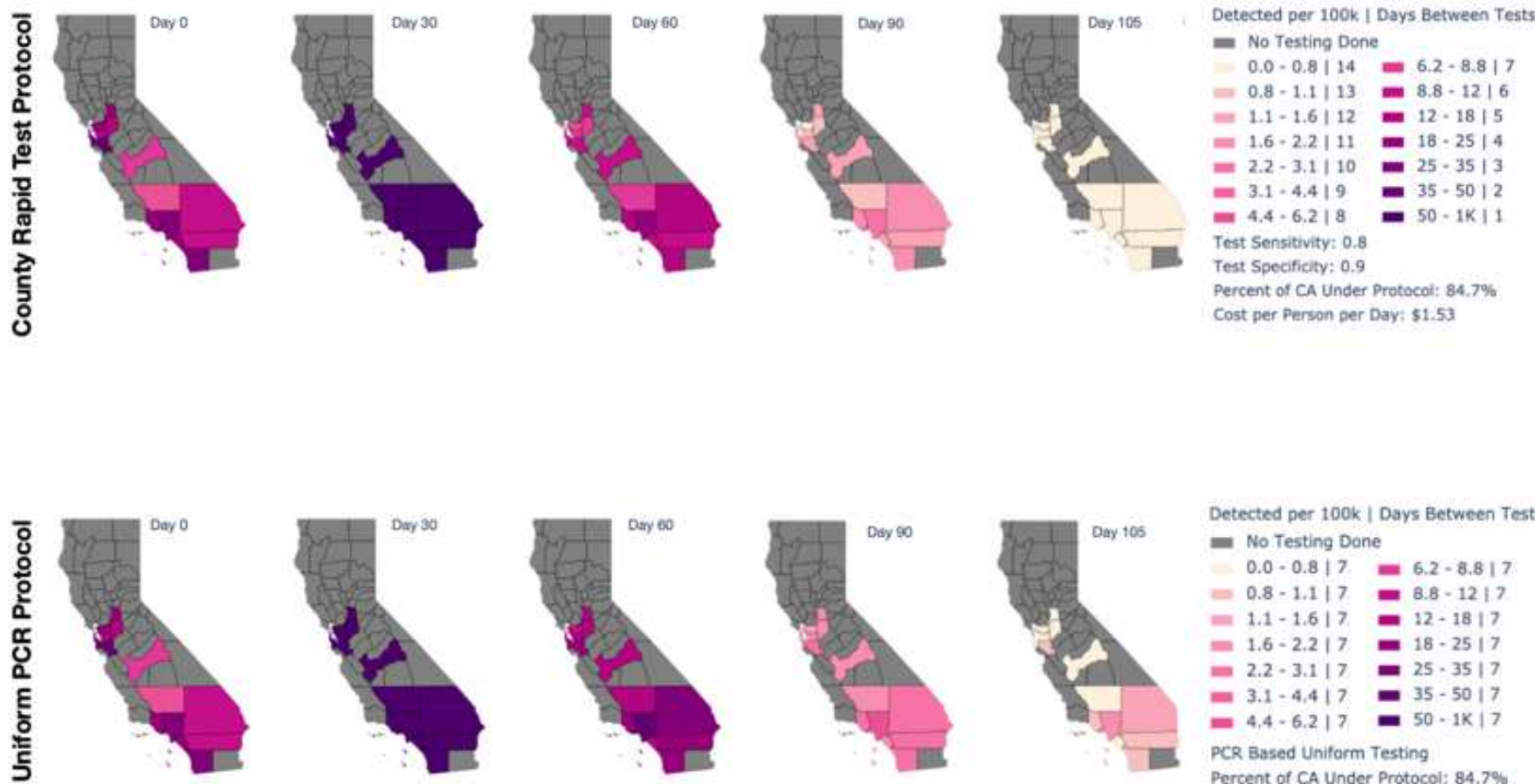

Detected per 100k | Days Between Tests $=$ No Testing Done

$0.0-0.817$

$=0.8-1.1 \mid 7=8.8-1217$

$=1.1-1.6|7=12-18| 7$

$=1.6-2.217=18-2517$

$=2.2 \cdot 3.117=25 \cdot 3517$

$=3.1-4.417=35-50 \mid 7$

$=4.4-6.217=50-1 \mathrm{~K} 17$

PCR Based Uniform Testing

Percent of CA Under Protocol: $84.7 \%$ Cost per Person per Day: $\$ 14.27$

\section{Figure 4}

Effect of County Based Rapid Testing strategy on COVID-19 outcomes in California. This protocol varies testing frequency in accordance to the number of recorded cases; the threshold for number of active infections which, if reached, signals to commence everyday testing (the highest frequency considered). A Rapid Test with an $80 \%$ sensitivity and $90 \%$ sensitivity versus is used in this deployment strategy. Shown is the total cost per person per day versus the cumulative infections, maximum simultaneously 
hospitalized, and cumulative deaths with varied thresholds for all of CA is shown. The County Based Rapid Testing strategy is compared to uniform testing, which distributes the same number of total tests used in the county strategy, albeit evenly across each county. The effects of uniform testing are modeled for both a Rapid Testing protocol and a qRT-PCR protocol (A). The effects of County Based Rapid Test Protocol and Uniform PCR Protocol on active infected detected population over time in CA are shown (B). The legend denotes the thresholds at which testing frequency is determined, the testing frequencies, the percent of CA population under the strategy, and the cost per person per day.

\section{Supplementary Files}

This is a list of supplementary files associated with this preprint. Click to download.

- 11062020NatureCommSupplementaryMaterial.docx 